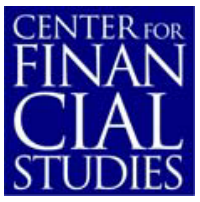

No. $2009 / 29$

Trust, Sociability and Stock Market Participation

Dimitris Georgarakos and Giacomo Pasini 


\section{Center for Financial Studies}

The Center for Financial Studies is a nonprofit research organization, supported by an association of more than 120 banks, insurance companies, industrial corporations and public institutions. Established in 1968 and closely affiliated with the University of Frankfurt, it provides a strong link between the financial community and academia.

The CFS Working Paper Series presents the result of scientific research on selected topics in the field of money, banking and finance. The authors were either participants in the Center's Research Fellow Program or members of one of the Center's Research Projects.

If you would like to know more about the Center for Financial Studies, please let us know of your interest.
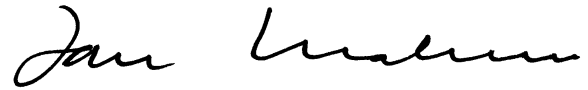

Prof. Dr. Jan Pieter Krahnen 
CFS Working Paper No. 2009/29

\title{
Trust, Sociability and Stock Market Participation*
}

\author{
Dimitris Georgarakos ${ }^{1}$ and Giacomo Pasini ${ }^{2}$
}

\author{
November 18, 2009
}

\begin{abstract}
:
We investigate the effects of both trust and sociability for stock market participation, the role of which has been examined separately by existing finance literature. We use internationally comparable household data from the Survey of Health, Ageing and Retirement in Europe supplemented with regional information on generalized trust from the World Value Survey and on specific trust to financial institutions from Eurobarometer. We show that trust and sociability have distinct and sizeable positive effects on stock market participation and that sociability is likely to partly balance the discouragement effect on stockholding induced by low generalized trust in the region of residence. We also show that specific trust in advice given by financial institutions represents a prominent factor for stock investing, compared to other tangible features of the banking environment. Probing further into various groups of households, we find that sociability can induce stockholding among the less well off in Sweden, Denmark, and Switzerland where stock market participation is widespread. On the other hand, the effect of generalized trust is strong in countries with limited participation and low average trust like Austria, Spain, and Italy, offering an explanation for the remarkably low participation rates of the wealthy living therein.
\end{abstract}

JEL-Classifications: A13, D12, D8, G11

Keywords: Trust, Sociability, Household Finance, Stockholding.

Georgarakos acknowledges financial support by the Center for Financial Studies (CFS) under the Research Program 'Household Wealth Management'. Pasini acknowledges financial support by the NETSPAR theme 'Pensions, savings, and retirement decisions' and from the University of Padua (research grant CPDA071899). We would like to thank Dimitris Christelis, Sergio Currarini, Ernst Fehr, Luigi Guiso, Michael Haliassos, Tullio Jappelli, Daniel Schunk, Stephan Siegel and seminar participants at the Annual Congress of the European Economic Association in Barcelona, the Annual Meeting of the German Finance Association (DGF) in Frankfurt, the MEA Conference on the Economics of Ageing in Deidesheim, the SHARE users conference in Mainz and at Venice University for useful suggestions. We are also grateful to Sven Fürth for excellent research assistance.

1 Department of Money and Macroeconomics, House of Finance, Grueneburgplatz 1 PF H32, 60323, Frankfurt am Main, Germany. E-mail: georgarakos [at] wiwi.uni-frankfurt.de; tel: +49 6979833830

2 Dipartimento di Scienze Economiche, Università Ca' Foscari, Cannaregio 821, 30121,Venezia, Italy. E-mail: giacomo.pasini [at] unive.it; tel: +390412349228. 


\section{Introduction}

Literature on the implications of various aspects of social capital on household portfolio decisions has rapidly developed. Guiso, Sapienza and Zingales (2004) show that Italian households tend to invest higher amounts in financial assets and to make less use of informal credit when they live in regions with high blood donation, electoral participation, and trust rates. Recently, Guiso, Sapienza and Zingales (2008, henceforth GSZ) have examined the effects of trust on stock market participation using Dutch and Italian survey data with information on a self-reported measure of trust. GSZ also associate differences in stock market participation across countries with variation in aggregate levels of trust by regressing the share of stockholders in each country on the average levels of trust and few other country-wide indicators (quality of legal enforcement and existence of a common law system). They show that countries with high prevailing trust exhibit on average high stock ownership rates, consistently with the fact that prospective investors in low-trust countries perceive a higher probability of being cheated.

In a related framework, Hong, Kubik and Stein (2004, henceforth HKS) provide evidence that sociability, as proxied by relationships with neighbors and church visits, fosters stock market participation. They mainly attribute this finding to the fact that 'word-of-mouth' information sharing lowers information costs, triggering participation of the more sociable individuals. As the authors stress: "While the social-capital variables used by Guiso et al. (2004) are obviously quite different from our social-interaction proxies, one might stretch and argue that our results reflect a similar kind of social-capital mechanism. [...] Although it is hard for us to address this hypothesis fully with our data, we can take a small step by looking at the effect of our social interaction variables on checking account use". Their presumption is that current accounts are subject to small entry costs and thus sociability, unlike social capital, should not play any role in their use. HKS derive an insignificant effect of their social interaction indicators on current account ownership that is interpreted as indirect evidence for the distinct role of sociability from other particular aspects of social capital like trust.

Trust and sociability might be positively associated but affect stock ownership via two different channels, as the aforementioned studies imply. Sociability serves to reduce fixed participation costs through cheaper information sharing. Mistrust lowers the expected return from an investment, given that potential investors need to take into account the possibility that a 
contract will not be respected by the counterpart. The distinct role of the two is also supported by literature on social capital that will be reviewed in the next section.

The key motivation of our study is to examine under the same framework the contributions of trust and sociability to stockholding and evaluate the possible implications for observed differences in households' investment behavior across European countries. To this end, we employ the standard portfolio model of GSZ, which takes into account the probability to be cheated, and we extend it by incorporating sociability coherently with HKS. We then obtain distinct predictions about the role of trust and sociability for stock investing. We empirically examine these predictions using internationally comparable survey data containing information on household sociability and asset holdings, supplemented with information on trust prevailing in each region ('generalized' referring to other community members and 'specific' referring to financial institutions). A noteworthy observation that one can make out of these data is that while households' median net wealth holdings are roughly comparable across groups of European countries in the sample, stock market participation rates exhibit remarkable differences. For example, in countries with widespread stock market participation (Sweden, Denmark, and Switzerland) households with below median wealth exhibit twice as high participation rates relative to their more than median wealth counterparts in low participation countries (Austria, Spain, and Italy).

As our study compares to HKS it takes into account, apart from sociability, the trust levels that households experience in the region they reside. With reference to GSZ we extend to the household level their cross country investigation on the relevance of generalized trust for aggregate stockholding by using internationally comparable survey data and exploiting withincountry variation in trust levels. Extending international comparisons in stockholding to the household level allows us to take into account, apart from country-wide indicators, the prevailing trust in the region of residence, individual sociability, and various socio-economic factors that affect households' investment behavior. Another novel aspect of this approach is that it allows to examine and compare the investment decisions of specific groups of households and to make appropriate quantitative statements. Furthermore, we shed light on the role of trust from a different perspective by considering specific trust in advice given by financial institutions and examining its implications for stock investing when beliefs about certain features of the banking environment are taken into account. In comparison to the part of GSZ analysis that investigates 
the influence of self-reported trust on stockholding decisions within a given country (Netherlands and Italy), we allow for an independent role of sociability and we use a regional-average rather than an individual-specific measure of trust. Our approach is consistent with the notion of "contextual" trust in the social capital literature, namely that trust is determined by the aggregate perceptions of all members in the community and thus individuals are "trust-takers" (Knack and Keefer, 1997). As it will be discussed later, such a measure is less likely to suffer from endogeneity bias.

This paper contributes to the existing literature in the following ways. First, we show that generalized trust and sociability have distinct and significant effects on stockholding and that their relative contribution changes with household wealth holdings as well as with average stock market participation rates and trust levels in the country. Second, specific trust in advice given by financial institutions represents a prominent factor for stock investing, over and above the influence of other features of the banking environment faced by households (e.g. costs required to change banks, access to formal credit and internet banking). Third, we shed more light on the striking differences in stockholding across Europe by linking them to the regional variation in prevailing generalized trust and household heterogeneity in sociability.

We use data from the Survey on Health, Ageing and Retirement in Europe (SHARE), that interviews households aged 50 and above across eleven countries. SHARE data represent a rich source of information on various demographics and asset holdings and contain a series of questions about households' social behavior (participation in voluntary activities, in educational training courses, in sport and political clubs and so on). We use answers to these questions to construct a household-specific measure of sociability. We supplement SHARE with information on generalized trust prevailing at different regions within each country that we recover from the World Values Survey (WVS), a cross national survey on values and norms. WVS asks households whether they think other people should be trusted or not. This information allows us to compute the fraction of people who trust in each region in a given country and subsequently assign this to SHARE respondents who live in the same region. In the same way we merge SHARE data with regional information on specific trust in the advice given by financial institutions as well as households' assessment of the quality of financial services that we recover from a special Eurobarometer module. In addition, we include in our dataset regional GDP 
growth rates and participation in EU elections from various data sources in order to perform a number of robustness checks.

We estimate net positive effects of both trust and sociability on stock market participation and we present some evidence suggesting that sociability can partly balance the discouragement effect on stockholding induced by low generalized trust in the region of residence. We find that more sociable households are more likely to invest in stocks compared to their less sociable counterparts and this effect is stronger in countries with widespread stock market participation. We also estimate independent positive effects on stockholding due to the regional variation in generalized trust as well as in trust specific to financial institutions that are net of the role of sociability, of various socio-economic characteristics, and of differences in institutional and other nationwide factors that are captured by country dummies in our models. Trust in the advice given by financial institutions in particular has a prominent role in stock investing, over and above the influence of regional disparities in the costs required to change banks and in the access to internet services.

The effect of generalized trust is significant in countries with low stock market participation rates and relatively low average trust and is particularly strong for the wealthy households. For example, we find that if a wealthy household living in a low trust region in Austria, Spain, and Italy moves to a region of higher generalized trust within these countries, other things equal, can nearly double the probability of investing in stocks directly. Thus, low prevailing generalized trust offers a possible explanation for the remarkably low participation rates among the better off who live in the aforementioned countries. On the other hand, living in a region with higher generalized trust does not contribute to an increase in stockholding in countries where stock market participation is widespread and the average trust level is high, like in Sweden, Denmark, and Switzerland. Nevertheless, higher sociability in these countries can foster direct stock market participation among the less wealthy households.

The rest of the paper is organized as follows. Section 2 reviews the existing literature and provides the theoretical justification in treating trust and sociability as distinct concepts. Section 3 presents the implications of considering both the probability to be cheated and sociability in a standard portfolio model. In section 4 we provide details on the data at hand and we present related descriptive statistics. Section 5 discusses the econometric specification, and section 6 reports the empirical results. Section 7 offers concluding remarks. 


\section{Trust and Sociability in the Social Capital Literature}

Trust and sociability are recurrent concepts in the social capital literature, both in Sociology and in Economics. Durlauf and Fafchamps (2004), after reviewing several relevant studies distinguished three common features: “(a) Social capital generates positive externalities for members of a group; (b) these externalities are achieved thanks to shared trust, norms and values and their effect on expectations and behavior; and (c) shared trust, norms and values arise from informal organizations based on social network and associations." Thus, it is difficult to reduce social capital to a single definition, and it is quite common in empirical investigations to focus on particular and measurable aspects of social capital. Islam, Merlo, Kawachi, Lindström and Gerdtham (2006) proposed a distinction into "cognitive" and "structural" social capital which is coherent with Durlauf and Fafchamps (2004) observations: the former is operationalized into people's perceptions about the level of interpersonal trust, sharing and reciprocity; the latter regards the density of social networks, or patterns of civic engagements.

We first concentrate on the economic rationale behind the association of cognitive social capital with stock market participation. La Porta, Florencio and Shleifer (2006) state that financial agreements require adequate level of transparency. The buyer must believe that the asset issuer (i.e. the financial institution or the Government) will be able to repay the agreedupon yield in the future; she must also be sure that the contract that she is writing is enforceable and covers all the relevant contingencies; moreover she should believe that in case of a litigation the attorney fees are bearable and the judicial process is fair and fast. Those conditions can be guaranteed by an appropriate set of securities laws or, as Guiso, Sapienza and Zingales (2004) suggest, by generalized trust. In a high-trust society informal agreement may substitute for costly contracts, litigations are less frequent, costs to protect from property rights violations are lower, and institutions, both private and public, are likely to be perceived as more credible. Knack and Keefer (1997) formalize this idea and define trust in terms of beliefs in a game theoretic framework: "[Trust is] the fraction of people in a society who expect that most others will act cooperatively in a prisoner's dilemma context". The intuition is that trust is a feature of the community an individual leaves in, and not a personal trait: a single member may be trustworthy towards his peers, but this feeling must be reciprocated by others in order to be rewarding. Thus, trust is determined by the aggregate perceptions of all the members, while single individuals are 
"trust-takers", meaning that no one can modify the overall level of trust perceived in a community. ${ }^{1}$

We measure the prevailing trust in a given region as the proportion of people in this region who state that trust the others. This is a quite common approach to measuring generalized trust and it has been used by Guiso, Sapienza and Zingales (2004), Knack and Keefer (1997) and by Rostila (2008) to assess the impact of trust on portfolio decisions, economic growth, and health outcomes respectively. In the same way we construct a measure of regional trust in the advice given by financial institutions that gives a direct insight into this specific aspect of trust. ${ }^{2}$

Structural social capital (i.e. the density of social networks) can also affect stock market participation. Jackson (2006) reviews the recent advances in the field focusing mainly on the role of information acquired through social interactions in determining human behavior and economic outcomes. ${ }^{3}$ Social interactions can serve as a mean of 'word-of-mouth' information diffusion or of observational learning, reducing information costs and lowering effectively the stock market participation hurdle (see HKS). For example, a prospective investor may learn from friends or acquaintances about high-return stocks or how to manage stocks efficiently. Therefore, "sociability" in our context is a measure of interactions with reliable and potentially informative acquaintances. Granovetter (1978) argues that a single connection with someone outside the restricted network of relatives and close friends can be more helpful in finding a job than a dense but isolated set of relations, i.e. what matters are "weak" ties as contrasted to "strong" ties. 4 Following HKS, we adopt an index that is consistent with "weak" ties and it is based on responses to survey questions regarding participation in political parties, sport and cultural clubs, charities and similar activities. This approach is quite common in the sociology literature (see Granovetter, 1983 and Putman, Leonardi and Nanetti, 1993). Measures of network centrality as in Calvó-Armengol, Patacchini and Zenou (2005) or network density as in Vega-Redondo

\footnotetext{
${ }^{1}$ The argument that trust should be studied as a collective phenomenon and not as a property of individuals has been also made in the sociology literature (see for example Newton, 2001).

${ }^{2}$ We use the terms 'trust' and 'generalized trust' interchangeably, while trust in the advice given by financial institutions is always termed as 'specific trust'.

${ }^{3}$ There is also a growing related literature of peer group effects on various economic decisions. See for example the works of Madrian and Shea (2000) and Duflo and Saez (2002) who show that individuals' decisions about their retirement investment plans are influenced by the choices of their working colleagues.

${ }^{4}$ Such a distinction resembles the one between "bridging" and "bonding" social capital, proposed by Putnam (2000). "Bonding" social capital refers to the relationships within homogenous groups, i.e. the links within a family, among relatives or close friends. "Bridging" social capital refers to the ties among those who belong to different ethnic or religious groups, or among individuals with different educational and occupational background.
} 
(1996), which are widely used in economics due to their theoretical link to network theory, do not fit in our setup given that they measure characteristics of "strong" ties networks.

\section{Trust and Sociability in a Standard Portfolio Model}

In order to illustrate in an analytical framework the distinct roles of trust and sociability, we employ the portfolio model presented by GSZ and we extend it in order to account for sociability in a way coherent with HKS. There are two assets available to investors: a safe asset, which yields return $r_{f}$, and a risky asset with an uncertain return $\widetilde{r}$ distributed with mean $E[\widetilde{r}]=\bar{r}>r_{f}$. Given an initial wealth $W$, an individual who invests a positive share of her wealth $\alpha$ in the stock market maximizes the following expected utility function:

$$
\left.\max _{\alpha} E U \mid \alpha \tilde{r} W+(1-\alpha) r_{f} W\right\rfloor
$$

Therefore, she will choose to participate in the stock market (i.e., $\alpha>0$ ) if

$$
E U\left[\alpha \tilde{r} W+(1-\alpha) r_{f} W\right\rfloor \geq U\left\lfloor r_{f} W\right\rfloor
$$

While $\widetilde{r}-r_{f}$ measures the return from bearing market risk, the potential investor may face risk factors that are not accounted in $\widetilde{r}$ : for example, the recent default of Lehman Brothers was clearly unanticipated and was not reflected in its market valuation. The stock investment can also become worthless if the contract signed is not enforceable (e.g. because the intermediary goes bankrupt or the broker runs away with money) or if costs are unbearable in case of litigation. Thus, we define mistrust as the probability $p$, independent from the probability distribution of the risky asset return, that individuals assign to the event that the value of a planed investment (initial capital and interest) goes to zero. In the previous section we described trust as a feature of the community an individual belongs to. Thus, we treat $p$ as common to all the individuals living in the same region. It actually serves as a discount factor that a prospective investor applies to her utility function. This is an inherently partial equilibrium framework: individuals are "trust 
takers" i.e. each of them behaves as her choice had no effect on the equilibrium level of $p$. The new participation condition is given by:

$$
\left.(1-p) E U\left\lfloor\alpha \tilde{r} W+(1-\alpha) r_{f} W\right]+p U\left[(1-\alpha) r_{f} W\right] \geq U \mid r_{f} W\right\rfloor
$$

It is apparent from various survey data that many households do not participate in the stock market and fixed participation costs have been proposed as a leading explanation for this trend (Mankiw and Zeldes, 1991, Haliassos and Bertaut, 1995, Vissing-Jorgensen, 2003). Such costs include not only brokerage and monetary fees but also non-tangible costs such as costs of time, costs of processing information as well as costs of picking and monitoring advisors and keeping up with market developments.

Following GSZ, we introduce a fixed cost of participation $f$ : if individual $i$ decides to enter the stock market, she has to pay an entry fee and then to allocate the remaining disposable wealth $W-f$ between the safe and the risky asset. Alternatively, she can decide not to invest in stocks, saving the participation cost. Hence, the participation condition becomes:

$$
(1-p) E U\left[\alpha \widetilde{r}(W-f)+(1-\alpha) r_{f}(W-f)\right\rfloor+p U\left[(1-\alpha) r_{f}(W-f)\right\rfloor \geq U\left\lfloor r_{f} W\right\rfloor
$$

implying that for each given level of mistrust $p$, the introduction of $f$ lowers participation.

Sociability allows individuals to reduce the fixed participation costs through cheaper information sharing and effectively augments the disposable wealth, which in turn induces participation. Following HKS we assume that the fixed participation cost for an individual can be specified as follows:

$$
f=f(\pi) \text { where } f(0)=\bar{f}, d f(\pi) / d \pi<0, \lim _{\pi \rightarrow \infty} f=\underline{f} \text { where } \underline{f} \geq 0
$$

$f$ is bounded between $\underline{f}$ and $\bar{f}$ and it is decreasing in $\pi$, the number of people who invest in stocks among individual's acquaintances. This is consistent with the notion that the cost of participation for an individual is reduced when more of her peers participate. As already explained, the network serves as a mean of 'word-of-mouth' information diffusion. A sociable individual has a 
larger social circle and thus she is more likely to meet people who have invested in the stock market and thus can convey valuable information about stockholding. It should be noted that $\pi$ represents the total number of peers who participate, and not the fraction of people who participate: a more sociable individual, even if she takes part to activities that are not directly related to asset investment, is likely to meet a larger number of people who participate in the stock market than her non-sociable counterparts. Therefore, each individual chooses $\alpha$ in order to maximize her expected utility conditional on trust, sociability, wealth, and rates of return:

$\max _{\alpha} E U\left[\alpha \mid p, \pi, r_{f}, \widetilde{r}, W\right]$
where $E U[\alpha]=\left\{\begin{array}{cc}(1-p) E U\left[\alpha \widetilde{r}(W-f(\pi))+(1-\alpha) r_{f}(W-f(\pi))\right]+ & \text { if } \alpha>0 \\ p U\left[(1-\alpha) r_{f}(W-f(\pi))\right] & \text { if } \alpha=0 \\ U\left[r_{f} W\right] & \end{array}\right.$

Then, the participation condition is written as:

$$
(1-p) E U\left\lfloor\alpha \widetilde{r}(W-f(\pi))+(1-\alpha) r_{f}(W-f(\pi))\right\rfloor+p U\left[(1-\alpha) r_{f}(W-f(\pi))\right\rfloor \geq U\left\lfloor r_{f} W\right\rfloor(7)
$$

Given that market risk is treated as in standard portfolio models and the introduction of trust and sociability neither affects the properties of the utility function nor the distribution of risky return $\widetilde{r}$, we can abstract from market risk and re-define equation (7) in terms of $\hat{r}$, the certainty equivalent of $\tilde{r}$ :

$$
\left.(1-p) U\left[\alpha \hat{r}(W-f(\pi))+(1-\alpha) r_{f}(W-f(\pi))\right\rfloor+p U\left[(1-\alpha) r_{f}(W-f(\pi))\right\rfloor \geq U \mid r_{f} W\right\rfloor
$$

The main difference from GSZ model is the fact that the participation cost $f$ is no longer constant but it is now assumed to decrease in sociability $\pi$. This implies that more sociable individuals dispose of a higher wealth endowment to invest in the stock market. More formally, the first empirical implication of the model is that for any value of $p$, the left hand side of (8) is monotonically increasing in disposable wealth $(W-f(\pi))$ and thus it is monotonically increasing in $\pi$. Consequently, the probability of participation (i.e. when condition (8) is 
satisfied) is increasing in $\pi$ given the level of trust (1-p). Note that $\pi$ represents a peer effect, and as such it may act as a social multiplier on wealth (and potentially on other determinants of participation omitted from this simple model $)^{5}$ : this feature of the model may lead to multiple equilibria. Establishing the set of conditions on $U$ that rule out multiple equilibria is beyond the scope of this paper.

The second empirical implication of the model derives from proposition 3 of GSZ. Define $A=\alpha \hat{r}(W-f(\pi))+(1-\alpha) r_{f}(W-f(\pi))$ and $B=(1-\alpha) r_{f}(W-f(\pi))$. Since we assumed $E[\widetilde{r}]>r_{f}>0$, as long as $W>f(\pi)$

$\left.U \mid \alpha \hat{r}(W-f(\pi))+(1-\alpha) r_{f}(W-f(\pi))\right\rfloor>U\left\lfloor(1-\alpha) r_{f}(W-f(\pi))\right\rfloor$

Therefore, for a given $\pi,(1-p) U[A]+p U[B]$ is decreasing in $p$ : the probability of participation is increasing in the level of trust (1-p).

The above suggest that trust and sociability have distinct effects on stock market participation when the standard portfolio model of GSZ that takes into account trust is extended to incorporate sociability coherently with HKS. This is not the only implication we can obtain from the model: the left hand side of equation (8) is a linear combination of continuous utility functions, thus if there exists a solution $\alpha^{*}$ to the maximization problem with $p>0$ and $0<\pi<\infty$, then it must exist a triplet ( $W=\underline{W}, p=\bar{p}, \pi=\underline{\pi}$ ) such that (8) holds with equality:

$$
(1-\bar{p}) U\left[\alpha \hat{r}(\underline{W}-f(\underline{\pi}))+(1-\alpha) r_{f}(\underline{W}-f(\underline{\pi}))\right]+\bar{p} U\left[(1-\alpha) r_{f}(\underline{W}-f(\underline{\pi}))\right]=U\left\lfloor r_{f} \underline{W}\right\rfloor(10)
$$

Equation (10) implies that there exists a minimum value of wealth $\underline{W}$, a maximum value of mistrust $\bar{p}$, and a minimum value of sociability $\underline{\pi}$ for which a potential investor is indifferent between paying the fixed entry cost upfront and investing a fraction $\alpha$ (such that $0<\alpha \leq \alpha^{*}$ ) of the disposable wealth $W$ - $f$ in stocks on the one hand, and staying out of the market avoiding the fixed cost and investing all her wealth $W$ in the safe asset on the other hand. We can now

\footnotetext{
${ }^{5}$ Informally, the mechanism can be described as follows: a marginal increase in wealth of individual $i, W_{i}$, could induce $i$ to participate. This creates externalities for her peers and therefore affects their chances to participate. Thus the fraction of $i$ 's peers who participate $\pi_{i}$ is likely to increase itself, reinforcing the effect of a marginal increase in $W_{i}$.
} 
examine the interrelationship among $\bar{p}, \underline{\pi}$ and $\underline{W}$, the values of mistrust, sociability, and wealth respectively that trigger participation. Provided that the utility function is well behaved, the following proposition holds:

Proposition 1: Given $\alpha>0$ and $W=\underline{W}$, the level of trust $(1-\bar{p})$ and the minimum sociability level $\underline{\pi}$ which trigger participation are inversely related.

This is proved in appendix I using the implicit function theorem. Moreover, once sociability is fixed, proposition 4 by GSZ holds: "for any probability [...] $p$ there exists a wealth threshold $W(p)$ that triggers participation and $W(p)$ is increasing on $p$ ".

Based on the above, we perform a series of comparative statics exercises and the implications we draw can be summarized as follows:

i. Sociable households should have a higher probability to invest in stocks, because of their interactions with other investors. Thus, given mistrust $p$ and wealth $W$, the marginal effect of sociability should be higher in countries where there is a high density of stockmarket participants. This is in line with the HKS model predictions and consistent with the notion that in regions where only a minority invests in stocks even the sociable individuals will have few informative acquaintances in their social circle.

ii. Given $\alpha^{*}$ and $\pi$, the higher the wealth is, the lower is the trust threshold $(1-\bar{p})$ that triggers participation and vice versa. Thus, among the wealthy, we expect to observe a significant effect of a marginal increase in trust even in low trust communities, while this effect should not be present among the less wealthy. The same implication derives from the GSZ model.

iii. Given $\alpha^{*}$ and wealth $W$, participation can be triggered by a low level of trust $(1-\bar{p})$ if sociability $\underline{\pi}$ is high enough and vice versa.

In our empirical investigation we find evidence in favor of $\mathrm{i}$ and ii. As regards iii, our findings based on the pooled sample of countries suggest that sociability has a stronger effect on stockholding in low trust regions compared to its effect in high trust regions. Yet, the intensity of the relative effects of generalized trust and sociability varies across different countries or groups

\footnotetext{
${ }^{6}$ For a formal proof refer to appendix I and to GSZ.
} 
of households (e.g. sociability should be more effective in countries with high stockownership; trust can be more relevant for the wealthy). Thus, we assess empirically their relative impact on stockholding by examining various population subgroups.

\section{SHARE and WVS data}

We use data from the first wave of SHARE which was conducted in 2004 . $^{7}$ SHARE is a multi-disciplinary, cross-national survey that is representative to the population aged 50 and over. The survey took place in eleven European countries, namely Sweden (SE), Denmark (DK), Germany (DE), the Netherlands (NL), Belgium (BE), France (FR), Switzerland (CH), Austria (AT), Italy (IT), Spain (ES), and Greece ${ }^{8}$. The unit of analysis is the household, given that most of the asset questions are asked at household level. ${ }^{9}$ The common design of the survey has enabled international comparisons of household wealth holdings (see Christelis, Georgarakos and Haliassos, 2008).

SHARE contains all the necessary information to construct a sociability indicator similar to the one proposed by HKS. A household is classified as sociable if at least one of the partners took part to one (or more) of the following social activities the month preceding the interview: voluntary or charity work; educational or training course; a sport, social or other kind of club; activities organized by a political or community organization. ${ }^{10}$

The measure for generalized trust is obtained from the WVS using the same question that GSZ employ to calculate country wide trust rates. WVS is a collection of surveys across more

\footnotetext{
${ }^{7}$ This paper uses data from SHARE 2004 wave 1, release 2.0.1. SHARE data collection in 2004-2007 was primarily funded by the European Commission through its 5th and 6th framework programs (project numbers QLK6-CT2001- 00360; RII-CT- 2006-062193; CIT5-CT-2005-028857). Additional funding by the US National Institute on Aging (grant numbers U01 AG09740-13S2; P01 AG005842; P01 AG08291; P30 AG12815; Y1-AG-4553-01; OGHA 04-064; R21 AG025169) as well as by various national sources is gratefully acknowledged (see $\mathrm{http}: / / \mathrm{www}$.share-project.org for a full list of funding institutions).

${ }^{8}$ Greece is not included in the analysis due to difficulties in merging with WVS data. More details on data issues are provided in appendix II.

${ }^{9}$ The raw data consist of about 17,000 households, either couples or singles. We exclude households with nonresponding partners.

${ }_{10}$ The survey also asks about participation in activities organized by religious organizations. However, in some countries, due to differences in translation, the question was asked with reference to participation in church services. Given the inconsistent way that this question was asked, we do not consider it in the list of the social activities.
} 
than 60 countries that provide information about social norms and peoples' beliefs. ${ }^{11}$ Respondents are asked the following question:

"Generally speaking, would you say that most people can be trusted or that you need to be very careful in dealing with people?"

1. 'Most people can be trusted'

0. 'Can't be too careful'

In both SHARE and WVS we know the region of residence of respondents. Thus, we first calculate region-level averages based on responses to the above trust indicator from WVS and then assign the relevant average to every SHARE respondent who lives in the same region. In the same way we supplement SHARE data with information on regional GDP growth rates and participation in EU elections that we employ in a number of robustness checks. ${ }^{12}$

It should be noted that the regional average trust is computed over the sample of all adults in WVS, and it is not restricted to those aged 50 and above. This is consistent with the contextual nature of our trust indicator: a more than 50 years old individual (represented in the SHARE sample) interacts with adults of any age in her region of residence. Therefore, merging the two datasets allows us to employ a trust indicator that is fully coherent with the notion of "contextual" trust discussed in Section 2. ${ }^{13}$

The different nature of our trust and sociability indicators (contextual versus householdspecific) implies a different interpretation of an assumed "marginal increase" in the underlying variables. As regards the former, the hypothetical experiment corresponds to moving an individual, keeping unchanged her characteristics, from a region of given prevailing trust into another region with relatively higher trust. For sociability, the comparison is between an individual who is engaged in social activities and her non-social counterpart living in a region with the same prevailing trust.

Our analysis focuses on households' decision to participate directly in the stock market. SHARE offers straight information on direct stock ownership that enables international comparisons, abstracting from country differences in the availability of financial products that

\footnotetext{
${ }^{11}$ We used the European and World Values Surveys four-wave integrated data file, 1981-2004, v.20060423, 2006. Surveys designed and executed by the European Values Study Group and World Values Survey Association. File Producers: ASEP/JDS, Madrid, Spain and Tilburg University, Tilburg, the Netherlands. File Distributors: ASEP/JDS and GESIS, Cologne, Germany.

${ }_{12}$ More details about the data sources and the construction of the trust measure can be found in appendix II.

${ }^{13}$ As a robustness check, we have experimented with regional trust averages computed across WVS respondents more than 40 and 50 years old and the results are qualitatively similar to those we report.
} 
allow indirect investments in stocks. It also makes possible comparisons with GSZ who examine the association between average trust rates and the fraction of individuals owning stocks directly across countries. SHARE asks households who own mutual funds whether they are mostly invested in stocks, bonds or split between the two. Based on this information we have constructed a proxy of indirect stockholding by classifying each mutual fund holder as a stock owner. In section 6.2, apart from our baseline findings on direct stockholding, we also discuss the quantitative implications of our trust and sociability indicators with respect to this broader form of stockholding across various groups of households.

Figure 1 reports ownership rates of directly held stocks by country. It is immediately evident that there is significant variation across Europe: more than one out of three Swedish and Danish households invest in stocks directly, while this fraction goes down to 5\% in Spain. Figure 2 illustrates country averages of sociability and generalized trust. The two measures display considerable heterogeneity across countries and there is not an obvious trend suggesting any systematic relationship between the two.

Figure 3 uses a map to depict differences in regional trust levels across Europe. Regions are divided into 6 classes: the light blue regions have an average trust below $20 \%$, while in the darkest ones trust rates exceed $60 \%$ (each class in between covers a $10 \%$ range). Trust levels by region display a significant within-country variation. ${ }^{14}$

\section{Econometric Specification}

We estimate the following binary choice model which corresponds to a reduced form specification of model (8):

$y_{i}=\mathbf{x}_{i}^{\prime} \boldsymbol{\beta}+\gamma_{1}$ trust $_{j}+\gamma_{2} \pi_{i}+v_{i}$

where $y_{i}=\left\{\begin{array}{l}1 \text { if } U(A)>U(B) \\ 0 \text { otherwise }\end{array}\right.$, and $v_{i} \sim N(0,1)$

That is, $y_{i}$ is a dichotomous variable taking the value 1 if household $i$ owns stocks (i.e. utility from stock market participation, as described in section 3, is greater than utility in case of non participation) and 0 otherwise. We include under $\mathbf{x}_{i}$ a rich array of demographic and pecuniary characteristics that will be discussed in detail later. The variables of interest are $z_{i}$, a

\footnotetext{
${ }^{14}$ Such heterogeneity has been justified for Italy in the seminal work of Putnam, Leonardi and Nanetti (1993).
} 
dummy showing engagement in social activities that we use as a proxy for the unknown $\pi_{i}$ (i.e. the total number of peers who participate in the stock market) and trust $j_{j}$, that represents the fraction of trusting individuals in the region of residence $j$ of household $i$.

As discussed, we construct a sociability indicator that is similar to the one employed by HKS. It should be noted that sociability is exogenous to stock market participation as long as we assume that a stockholder does not decide to engage in social activities in order to acquire information about the stock market. Given the type of activities we consider (voluntary work, participation in sport, social, political clubs or training courses) this seems a plausible assumption. $^{15}$

Still, one might argue that the sociability indicator partly reflects the influence of other traits like pessimism, attitudes towards risk, health or cognitive limitations that are associated with engagement in social activities and are also likely to affect the propensity to invest in stocks. First, SHARE data offer details on various household characteristics and attitudes that we incorporate in our estimation in order to rule out the possibility that our sociability indicator picks up the effect of these traits. As it will be discussed later, we take into account, apart from standard demographic characteristics and resources, the influence of depression, objective and subjective health indicators, as well as cognitive abilities. In addition, we verify the robustness of our findings when an indicator of willingness to assume more than average financial risk is taken into account.

Second, a direct implication deriving from the assumed influence of sociability on stock market participation, is that the marginal effect of sociability should be higher in areas with widespread stockholding. This is the case given that a sociable household living in these areas is more likely to meet many acquaintances with useful information about stock investing, reducing effectively the participation costs that this household faces. At the other extreme, the estimated effect of sociability should be smaller in areas where only few have invested in stocks, provided that a sociable prospective investor is quite unlikely to find an informative acquaintance in her social circle. Results from our analysis, presented in section 6.2, offer empirical support to the

\footnotetext{
${ }^{15}$ As HKS stress, even if information on $\pi$ was directly available, the inference would be subject to the well-known "reflection problem" (i.e. had to be drawn from the estimated effect of the average participation of a given group on the participation choice of an individual belonging to this group) - see also Manski (1993). Instead, $z_{i}$ circumvents the issue of reverse causality, while it is natural to assume that an individual engaged in social activities is more likely to meet - other things equal - a larger number of people who participate in the stock market than her nonsociable counterpart.
} 
above premise. As it is stressed by HKS this fact strengthens the case that the sociability indicator picks up the real influence of social interactions and not that of personal traits that have not been adequately accounted for. That is, if the sociability indicator mostly reflects the influence of such personal traits, there is not any a priori reason to expect a differential effect of this indicator across areas with different prevalence of stockholding.

As regards the indicator of trust, the recent work by Fehr (2009) has raised concerns about the causal role of trust on various economic outcomes. The author argues that self-reported trust, as it is measured in survey data, is partly shaped by own beliefs about other people's trustworthiness which in turn have been influenced by formal and informal institutions. More generally, prevailing trust in a country can be partly determined by interactions with the institutional environment. For example, policies introduced to promote transparency in a country's stock market may have contributed to an increase in an individual's trust and in general trust in the population. Given the obvious difficulties in finding valid instruments that will correlate with trust but not with economic outcomes, Fehr suggests that such instruments can be obtained through laboratory experiments. ${ }^{16}$ Our measure for trust implicitly assumes that every individual residing in a given region is "trust taker". That is, a change in her trusting behavior or in her beliefs about others' trustworthiness cannot alter the overall trust level prevailing in this region. ${ }^{17}$ Moreover, cross country differences in average trust that are most likely related with country wide differences in policies and institutions or in aggregate beliefs, will be absorbed by the country dummies in our model. Thus, in the light of the above arguments our measure of trust as it compares to those that have been broadly used (either individualspecific at the micro level or country-specific at the macro level) is less likely to suffer from endogeneity bias almost by construction. Furthermore, we perform various robustness checks to ensure that our regional trust indicator does not incorrectly pick up effects that are due to regional differences in development, access to informal and formal borrowing, banking environment, and use of internet services.

\footnotetext{
${ }^{16}$ The author proposes to induce exogenously in one group optimistic beliefs and in another group pessimistic beliefs about other people's trustworthiness and subsequently examine the extent to which they result in divergent economic behavior.

${ }^{17}$ The concept can be seen as similar to considering individual consumers as price takers.
} 
We examine household participation in direct stockholding by estimating (11) by probit models. ${ }^{18}$ Given that our measure of trust is regional invariant, the estimated standard errors are corrected for clustering at regional level (see Moulton, 1990). In addition, we take into account the fact that missing values have been imputed in SHARE using a multiple imputation method. Hence, we perform the estimation and compute standard errors corrected for clustering within each implicate, and then combine the estimates and standard errors across implicates using the rules described in Rubin (1987). The estimated coefficients from binary choice models are not directly interpretable, thus we calculate and report marginal effects averaged across individuals using calibrated survey weights.

All our specifications control, apart from sociability and trust indicators, for a rich set of household demographics and measures of resources. More specifically, we take into account a broad set of demographics like age, gender, marital status, and the number of children. Given that bequest planning can affect portfolio allocations we also condition on the self-reported probability to leave a sizeable bequest. We take into account labor status by distinguishing among those working, retired, and unemployed. In addition, we allow for an independent role of resources including separate controls for net total wealth and income through an inverse hyperbolic sine transformation that allows for non-linear effects of these variables (see Burbidge, Magee and Robb, 1988). Controlling for resources is dictated both by theory, with its emphasis on 'cash on hand' as a key determinant of asset investments, and by the need to avoid confounding the role of determinants of interest with that of wealth, when the latter is not controlled for in the regression. ${ }^{19}$

Households with health problems are discouraged from investing in stocks according to Rosen and $\mathrm{Wu}$ (2004). In order to take into account the effect of adverse health conditions we include as explanatory variables both a subjective (self-reported health) and an objective (number of limitations in activities of daily living - ADL) health indicator. Christelis, Jappelli and Padula (2009) using SHARE data found a significant role of cognitive abilities for stockholding. In our specification we employ their cognitive indicator, namely the respondents'

\footnotetext{
${ }^{18}$ See Guiso, Haliassos and Jappelli (2002) for previous studies on household stock market participation and Campbell (2006) for a review on issues related to household finance.

${ }^{19}$ In each specification we exclude from total net wealth the value of stocks in order to avoid endogeneity issues.
} 
ability to recall words correctly out of a list that is read to them by the interviewer. On top of recall ability we control for depression as a measure of pessimism. ${ }^{20}$

Finally, we include country dummies to capture country-specific factors that are likely to affect stockholding, such as the level of financial development, market transparency, the legal environment, as well as the differences in companies' default risks. Country dummies will also capture differences in the average levels of trust across countries that as it was discussed are most likely to be due to country differences in the institutional environment. This implies that our trust indicator which measures differences in the regional levels of trust represents a conservative estimate of the overall effect of trust on stockholding. Summary statistics and a detailed description of all variables used in the empirical analysis are presented in Tables AII.1 and AII. 2 in appendix II.

\section{Empirical results}

In what follows we first discuss empirical results from regressions on the full sample at hand as well as from a series of robustness checks that examine the sensitivity of our baseline findings. Next we examine the relative contribution of generalized trust and sociability to stockholding across countries with different participation rates and households with different wealth holdings.

\subsection{The Effects of trust and sociability on stock market participation}

\subsubsection{Generalized trust and sociability}

We model the probability of direct stocks investments as a function of a broad set of household socioeconomic characteristics and of combinations of our generalized trust and sociability indicators. Table 1 presents average marginal effects and their standard errors from a series of probit regressions. In specification 1 we control for trust without taking into account the influence of sociability and vice versa in specification 2. In specification 3 we control for both,

\footnotetext{
${ }^{20}$ Characteristics in the case of couples represent a combination of the information from the two partners. In particular we use average age, worse reported health status, total number of limitations in daily activities and the maximum of: educational level, recall abilities and depression. Furthermore, the household is determined to be in the labor force if any of the two partners is working.
} 
while in specification 4 marginal effects have been computed from a model that includes an interaction term between trust and sociability. ${ }^{21}$

Marginal effects from the above specifications suggest an independent, economically important, and precisely estimated role for trust and sociability. More social households are 3.1 percentage points (pp) more likely to invest in stocks and this effect remains unchanged when regional trust is taken into account (it slightly increases to $3.6 \mathrm{pp}$ when interactions with trust are allowed). ${ }^{22}$ On the other hand, living in a region where a higher fraction of people trust (we assume a $15 \mathrm{pp}$ increase in regional trust which roughly corresponds to one standard deviation of this variable) is associated with a $2.1 \mathrm{pp}$ increase in the probability to own stocks directly. The above effects are net of various demographics, household resources, and country wide differences and are economically important given that the average participation rate in our sample does not exceed $13 \%$.

Based on specification 4 that allows for an interaction between our trust and sociability indicators we have also calculated the marginal effect of sociability within low and high trust regions. ${ }^{23}$ In low trust regions sociability increases by $5.1 \mathrm{pp}$ (s.e. .01) the probability to invest in stocks, while in high trust regions by $3.4 \mathrm{pp}$ (s.e. .007). This suggests that sociability can partly balance the negative effects on stockholding that are associated with living in a low trust region. Yet, the relative contribution of trust and sociability will be examined in detail in the next section where we investigate the stockholding choices across specific groups of countries and households.

The estimated effects of other covariates (education, health, financial resources, recall ability) display the expected signs and are in line with findings from existing literature on determinants of stockholding behavior. With reference to country dummies, estimated marginal effects are sizeable and consistent with patterns of stockholding rates across countries suggested by the raw statistics. ${ }^{24}$

In Table 2 we present marginal effects from various probit regressions in order to examine the sensitivity of our findings on trust and sociability. To ease comparisons

\footnotetext{
${ }^{21}$ Brambor, Clark and Golder (2005) point to frequent problems in empirical literature due to misspecification of models that include interaction terms and to the calculation of meaningless marginal effects. We follow their approach in calculating marginal effects for the two indicators of interest.

${ }^{22}$ The sample correlation of the two indicators is roughly 0.2 .

${ }^{23}$ We define as low (high) trust regions those where the fraction of people who trust the others is below (above) $24 \%(37 \%)$. These roughly correspond to the bottom and the top quartile of the distribution of regional trust rates.

${ }^{24}$ Country marginal effects are in comparison to Germany.
} 
specification 1 in Table 1 repeats estimated marginal effects from our baseline model that controls for both sociability and trust (specification 3, Table 1). We first examine the possibility that our regional-based trust indicator reflects regional differences in development. Our baseline model already controls for ppp-adjusted income, financial and real wealth per household and has taken into account average country differences in development through the country dummies. Yet, it may be argued that regional differences in development rates are not fully captured and are partly picked up by our measure of trust. To rule out such a possibility we computed average annual GDP growth rates between 2000 and 2004 by region and we include it as an additional regressor. This inclusion does not alter our findings on trust. The implied effect of regional differences in GDP growth rates, net of ppp-adjusted household resources and average crosscountry differences in development, is negative. This may suggest that the fast development of some European regions the five years preceding the survey was not accompanied by an analogous increase in household investment in stocks.

Due to data limitations our baseline specification does not include any control on households' attitudes towards risk and our estimates on trust and sociability may partly reflect such omitted information. The relevant information is surveyed in the 2006 SHARE wave for the first time and given the panel nature of the survey we retrospectively assign it to households interviewed in $2004 .{ }^{25}$ Then in specification 3 we include a dummy variable representing households who are willing to assume more than average financial risks in anticipation of higher returns. The estimated effect on this dummy has the expected sign, it is very precisely estimated, and suggests a sizeable impact. Yet, our estimated effects on trust and sociability remain unaffected when households' risk attitudes are taken into account. Next, in specification 4 we control for both regional GDP growth rates and households' willingness to assume more than average financial risks and the results remain unchanged.

As an additional robustness check we also experiment with a completely different indicator that has been employed by studies that examine the role of social capital on economic outcomes (e.g. Guiso, Sapienza and Zingales, 2004). If indeed sociability affects stockholding through an independent channel then it should not be affected by the use of such an alternative indicator, while the latter is likely to have an independent positive influence on stockholding. In

\footnotetext{
${ }^{25}$ The variable takes the value 1 if one of the partners in the households reports to be risk averse. Due to attrition between the two waves our sample drops to 9,919 observations.
} 
particular, we use information on voters' participation in 2004 EU elections by region that represents a broader, compared to the WVS question on trust, indicator of social capital. ${ }^{26}$ Results are shown in specification 5 of Table 2. Living in a region where a higher share of people participate in voting (we assume a $20 \mathrm{pp}$ increase in participation rates which roughly corresponds to one standard deviation of this variable) implies a $4.7 \mathrm{pp}$ increase to the probability of holding stocks directly. Notably the effect of sociability remains unchanged.

The above results provide us with confidence that trust and sociability have quite distinct and significant influence on stockholding behavior. However, their relative importance may vary across different groups of countries and/or households. We probe further into the relative contributions of generalized trust and sociability in stockholding across various groups of households in section 6.2.

\subsubsection{Trust in financial institutions, sociability, and the banking environment}

In this section, we employ data from Eurobarometer, a survey frequently conducted across EU countries to measure the views of European households on a broad range of socioeconomic issues. More specifically, we employ data from Eurobarometer 60.2, which was conducted at the end of 2003, and asks respondents details about the quality of various financial services they make use of. This allows us to probe further into the role of prevailing trust by examining the significance of a specific aspect of trust that refers to advice given by financial institutions, while taking into account regional variation in households' assessment about certain features of the banking environment (e.g. access to credit, costs in changing banks, and use of internet banking services). In addition, we use data from Eurobarometer 56.1, conducted in the end of 2001, that offer information on the prevalence of informal borrowing. It should be noted that Eurobarometer does not include Switzerland, meaning that estimations presented in the current section are based on the sample of the nine EU countries.

Our trust indicator employed in the baseline specification and alternative models of section 6.1.1 was based on a widely used measure of generalized trust that is taken from WVS. Eurobarometer asks individuals explicitly whether they trust the advice given by financial institutions. In particular, they are asked to indicate whether they agree or not with the following statement:

\footnotetext{
${ }^{26}$ For Switzerland regional participation rates in 2003 national elections are considered.
} 


\section{I usually trust the advice given by financial institutions}

According to the data $58 \%$ of respondents tend to agree with this statement. The vast majority of respondents appear to have some experience with financial advice, given that roughly $80 \%$ of the owners of simple financial instruments such as bank or saving accounts state that they typically expect to get advice from financial institutions. Table 3 summarizes country averages of the Eurobarometer indices used in this section.

We first calculate the fraction of those in each region who tend to agree with the aforementioned statement and subsequently assign the relevant figure to every SHARE respondent who lives in the same region. The correlation of this measure with the measure of generalized trust, used in the baseline specification, is 0.3 suggesting that the two are positively associated but there are not identically distributed across regions.

This may not be surprising. Generalized trust accounts for the aggregate perceptions of all members in a given community, and thus it is likely to be deeply rooted and associated with an area's history, norms, and traditions. On the other hand, the specific trust measure refers to beliefs of community members about trustworthiness of the services offered by a well defined subset of the community, namely financial institutions. For example, average generalized trust is comparable across Austria, Spain, and Italy, which are countries with a catholic prevalence. On the other hand, Austria shares many common features of financial institutions with Germany and other central European countries. Thus, specific trust in financial institutions in Austria is likely to be closer to central European countries than to Mediterranean ones. The above imply that examining specific beliefs about trustworthiness in financial institutions and contrasting them with beliefs regarding other aspects of the banking environment can shed more light on the role of trust in stock investing.

Marginal effects from the model that takes into account regional variation in trust in financial institutions as well as household-specific sociability are presented in specification 1 of Table 4. Notably, estimated effects on both indicators of interest are comparable to those from the baseline specification.

Previous research has linked national differences in GDP growth rates to differences in country-average trust (Knack and Keefer, 1997) and has documented a negative association between trust prevailing in Italian regions and the extent of informal borrowing (Guiso, Sapienza 
and Zingales, 2004). To take into account such sources of heterogeneity at regional level, we include in our specification GDP growth rates by region as well as an indicator that intends to capture the prevalence of informal borrowing in each region. The latter is derived from Eurobarometer 56.1 which asks respondents to specify whether there is anyone outside their own household they could rely on in case they need to borrow money to pay an urgent bill or mortgage. ${ }^{27}$ Results are summarized in specification 2. Estimated effects on the indicators of interest remain unaffected, while the GDP growth rates suggest a similar pattern to the one obtained for the baseline specification in section 6.1.1.

The main differences in credit institutions and the banking environment are countryspecific and as such have been accounted for by the country dummy indicators. Nevertheless, we examine the possibility the effect of trust in financial institutions on stockholding to pick up regional differences in banking services within a given country. To this end, we utilize information on household beliefs about various features of the banking environment and the quality of banking services in each region. First, we calculate the fraction of people in each region who agree with the following statement:

\section{You can borrow as much as you like, there are no real checks}

This provides an indicator of unrestricted access to formal credit markets, based on households' assessment. It can be suggestive for regional disparities in the share of households that have successfully applied for a loan, credit policies adopted by commercial banks, procedures required to apply for a loan, or even advertisement and marketing of loan products. In addition, we calculate the fraction of those in each region who state that it is very difficult to change banks. ${ }^{28}$ This indicator is likely to reflect regional differences in costs related to banking services that are due to differences in the density of bank branches, the procedures required and the fees charged for opening a bank account as well as the intensity of competition among banks. Results from the specification that includes these last two indicators are presented in specification 3 of Table 4 . The estimates on the variables of interest remain essentially unaffected, while living in regions that it is very difficult to change banks reduces the probability of investing in stocks.

\footnotetext{
${ }^{27}$ Respondents are asked the following question: "If you needed to borrow money to pay an urgent bill, like electricity, gas, rent or mortgage, is there anyone you could rely on to help you, from outside your own household?."

${ }_{28}$ The following question is asked: "Do you believe it is very easy, fairly easy, fairly difficult or very difficult to change banks?",
} 
As a final robustness check we add to the above indicators an index of the fraction of people in each given region who answer affirmatively to the following question:

Have you ever used the Internet to make a bank transaction?

This is likely to represent not only regional differences in technological development and internet connections, but also easier - in terms of money and time - access to banking services. ${ }^{29}$ Results are presented in specification 4, Table 4. According to these, the higher the share of people in a region who have access to banking internet services, the higher the probability of investing in stocks.

In sum, results from this section suggest that trust with explicit reference to financial institutions affects stockholding significantly and independently from sociability, implying a similar effect to that derived for generalized trust. Furthermore, when regional heterogeneity in household beliefs about certain features of the banking environment is taken into account the role of trust in financial institutions remains economically significant. These differences in beliefs are most likely to reflect regional disparities in tangible costs of banking services. That is, better quality of the banking environment in a household's region of residence (as regards lower costs in changing banks and easier access to banking services) fosters stock investing, yet trust in financial institutions appears as a prominent factor for such decisions.

\subsection{Generalized trust and sociability across countries with different participation and trust rates and households with different wealth holdings}

As we pointed out in section 2, sociability facilitates easier access to information through 'word-of-mouth' information sharing. A sociable household is more likely - other things equal to acquire information about performance and efficient management of stocks in regions where a critical mass of people invests in the stock market. Thus, we anticipate sociability to have a stronger impact on stockholding in countries with higher participation rates. HKS find supporting evidence for this premise by estimating the effects of their sociability indicators in US states with low, medium, and high stock market participation rates.

We examine this notion by taking into account the influence of generalized trust and sociability across European countries with different participation rates. The group of high

\footnotetext{
${ }^{29}$ Using survey data from the US HRS, Bogan (2008) provides evidence that households that use the internet regularly are more likely to invest in stocks, given that internet facilitates access to financial information.
} 
participation countries consists of Sweden, Denmark, and Switzerland where more than $25 \%$ of households have invested in stocks directly. At the other extreme, we consider Austria, Spain, and Italy where participation rates are about 5\%. France, Germany, Netherlands, and Belgium represent the intermediate group with stock ownership rates around $15 \%$. The same country classification holds when one considers ownership of stocks held directly and through mutual funds. ${ }^{30}$ Thus, in what follows we discuss apart from the baseline findings on direct stock ownership, the quantitative importance of our trust and sociability indicators with reference to this broader form of stockholding.

When different groups of countries are examined one should also take into account in the interpretation of results the fact that median prevailing trust is different across these groups. The median regional generalized trust rates are $.32, .35$ and .58 in the low, medium and high participation groups of countries, respectively. ${ }^{31}$

In what follows we also explore differences in our trust and sociability indicators between more than median and less than median wealth households. According to GSZ low trust can offer an explanation for the limited stock market participation of wealthy households given that they face relatively small participation costs.

Figure 4 illustrates stockholding rates across the relevant subsamples. It becomes apparent that there are remarkable differences across Europe. For example, in high participation countries (Sweden, Denmark, and Switzerland) households with below median wealth exhibit twice as high participation rates, reaching $20 \%$, relative to their more than median wealth counterparts in low participation countries (Austria, Spain, and Italy). This difference is even more striking if one takes into account the fact that households' ppp-adjusted median net wealth holdings are comparable - as it will be shown below - across the three groups of countries.

In order to ease comparisons Table 5 summarizes marginal effects on sociability and trust indicators from eleven probit models estimated in different subgroups. ${ }^{32}$ Model D3 presents again results from our baseline specification that has been estimated over the original sample of households.

\footnotetext{
${ }^{30}$ The average participation rates in stocks held directly or through mutual funds are $47 \%, 23 \%$ and $7 \%$ in the high, medium and low participation countries, respectively.

${ }^{31}$ The standard deviations of the regional trust rates in our sample are $.07, .10$ and .13 for the low, medium and high participation groups of countries, respectively.

${ }^{32} \mathrm{We}$ estimate our baseline model in various groups of households across the high, medium, low participation countries and below and above median wealth groups using in each case the relevant country dummies.
} 
Results from models C3, B3, and A3 suggest that sociability has a proportionally stronger effect on stockholding when we consider groups of countries with a higher fraction of people participating in the stock market (the effect raises from $2.4 \mathrm{pp}$ in low participation countries to $4.6 \mathrm{pp}$ in high stock participation countries). The relevant effects for stocks held directly and through mutual funds (not reported) are $3.4 \mathrm{pp}$ and $8.2 \mathrm{pp}$ (both significant at 1\%) in low and high participation countries, respectively. ${ }^{33}$ As already discussed, in countries with higher participation rates more households are aware about stocks and therefore a prospective investor who is more sociable has higher chances to find a stockholder in her social circle.

As regards the role of trust, it significantly contributes to stockholding only in low participation countries (C3). As it was discussed this is the group of countries with the lowest median prevailing trust. The results suggest a $5.1 \mathrm{pp}$ higher probability to invest in stocks directly due to an assumed $15 \mathrm{pp}$ increase in the regional trust rates. We obtain the same picture as regards ownership of stocks and stock mutual funds with the estimated effect of trust in low participation countries being $8.2 \mathrm{pp}$ (significant at 1\%). Thus, our findings imply that living in a region with higher trust levels can induce stockownership only when the median trust level in the country is relatively low. Yet, differences in trust levels by region do not seem to matter when the prevailing level of trust in the country is generally high (A3).

When we split households by median net wealth levels, we find significant effects of trust only among those with more than median wealth (D1). On the other hand, the effect of sociability is positive and significant in both wealth subgroups (D1 and D2).

In order to probe further into the relative contribution of trust and sociability in stock market participation we split our sample in each of the three country groups into households with more than median and less than median wealth holdings. Median (non-stock) net wealth holdings are roughly 140,000, 150,000 and 130,000 euro (ppp-adjusted) for the groups of high, medium and low participation countries respectively. These amounts are comparable across the three country groups making more puzzling the significant gap in stockholding rates between the below median wealth households in high participation countries and their affluent counterparts in low participation countries.

\footnotetext{
33 The complete set of estimates on stocks held directly or through mutual funds are available from the authors upon request.
} 
Results from this sample split up give us additional insights on the role of generalized trust and sociability for stockholding. First, we estimate a very strong effect of trust among households with more than median wealth in low participation countries (C1). Our results suggest that if a wealthy household in Austria, Italy, and Spain moves to a region where trust rates are $15 \mathrm{pp}$ higher relative to the region in which is currently located will increase - other things equal - by $7.3 \mathrm{pp}$ the probability to invest in stocks directly. Given that participation rates among the more than median wealth households in these countries are around $8 \%$, living in a high trust region can nearly double the probability to invest in the stock market directly. The respective estimated effect for stocks held directly and through mutual funds is $10.2 \mathrm{pp}$ (significant at 1\%) that corresponds to an $80 \%$ increase relative to the existing ownership rates among the wealthy in low participation countries. On the other hand, trust does not affect stockholding in high stock participation and medium stock participation countries regardless of households' wealth holdings. Thus, our estimated effects on trust in the full sample (D3) or in the low participation countries (C3) are mainly driven by the influence of trust on the stockholding decisions of wealthy households in low participation countries.

Second, the strongest effect of sociability is estimated for the less than median wealth households in high participation countries (A2). A sociable household with below median wealth in Sweden, Denmark, and Switzerland is $5.3 \mathrm{pp}$ more likely to invest in stocks compared to a non-social counterpart. On the other hand, sociability does not influence stockholding among wealthy households in high participation countries. Finally, we estimate significant effects of sociability in medium participation countries for both wealth groups (B1 and B2) and in low participation countries only among the wealthy $(\mathrm{C} 1)$. When we examine ownership of stocks and stock mutual funds we find a similar pattern regarding the influence of sociability with the exceptions that the estimated effects among the wealthy in high participation countries and the less wealthy in low participation countries turn to be significant. ${ }^{34}$ Sociability may be more effective for the ownership of stocks and stock mutual funds given the higher - relative to direct stocks - prevalence of these investments.

All in all, results from this section suggest that the relative contribution of generalized trust and sociability on stockholding can vary across different groups of households. Sociability can induce stockholding among the less wealthy potential investors in Sweden, Denmark, and

\footnotetext{
${ }^{34}$ The estimated effect of sociability in the former group is $8.4 \mathrm{pp}$, and in the latter $2.8 \mathrm{pp}$, both significant at $1 \%$.
} 
Switzerland where a significant part of the population participates in the stock market. Yet, neither trust nor sociability can explain what triggers non participation in direct stocks among the wealthy in these countries. On the other hand, the effect of trust is strong in countries with limited participation and low country-wide median levels of trust like Austria, Spain, and Italy and regard in particular the wealthy households. Thus, low prevailing generalized trust is likely to offer an explanation for the remarkably low participation rates among the wealthy living therein. Finally, both indicators do not matter for direct stockholding among the less wealthy in low participation countries. These households may not even consider the option to invest in the stock market due to their ignorance about the existence of stocks (for example, Guiso and Jappelli, 2005 document such ignorance over a significant fraction of Italian households).

\section{Conclusions}

This paper aims to assess the implications of both trust and sociability for stock market participation, the role of which has been examined separately by the existing literature. HKS have established the independent role of sociability on stockholding, while GSZ have associated cross country differences in aggregate stock market participation with average trust levels. We use internationally comparable survey data from SHARE supplemented with regional information on generalized trust that we recover from WVS. The data allows us to investigate differences in stockholding across ten European countries and to link them to both regional variation in prevailing trust and household heterogeneity in sociability.

We show that trust and sociability play distinct roles for stock market participation in a context of a simple portfolio model that takes into account both the probability to be cheated and the reduction in participation costs through 'word-of-mouth' information sharing. In our empirical investigation we find strong support in favor of such a distinct role. As regards the relative influence of the two, a general prediction of this simple model is that a reduction in trust can be counterbalanced by an increase in sociability. In the full sample of data, we find some evidence supporting such a prediction. The above imply that trust and sociability should be taken both into account when studying households' stock market participation decisions.

In order to probe further into the relative contributions of trust and sociability to stockholding we examine groups of countries with different stock ownership, different prevailing 
trust, and households with different wealth holdings. The relevance of trust and sociability changes across subsamples that comprise different prospective investors. Sociability is found to induce stockholding mainly in Sweden, Denmark, and Switzerland where a significant part of the population participates in the stock market. In these countries median generalized trust rates are high and stockholding is not affected by differences in regional trust. On the other hand, regional variation in prevailing trust fosters stockownership in countries with limited participation and low average trust levels like Austria, Spain, and Italy. This is particularly the case for the better off households living therein: if a wealthy household that lives in a low trust region in the aforementioned countries moves to a higher trust region, other things equal, can nearly double the probability of investing in stocks directly.

We also supplement our data with regional information on specific trust in the advice given by financial institutions as well as households' assessment of the quality of financial services that we recover from a special Eurobarometer module. We show that specific trust to financial institutions affects stockholding significantly and independently from sociability, implying a similar effect to that estimated for generalized trust. Furthermore, specific trust in financial institutions appears as a prominent factor for stock investing, net of regional differences in households' assessment of certain features of the banking environment that are likely to reflect differences in tangible costs such as costs in changing banks, and in access to credit and internet banking services.

Our findings suggest that advancing competition among banks can contribute to stockholding by reducing costs required to change banks or by facilitating access to banking services. Yet, promoting trust in financial institutions through measures that ensure transparency in transactions or protect investors' rights should be a top priority if the policy objective is to foster stock investing. Results from this study are also likely to be of interest to policy makers who wish to promote stockholding among specific groups of households. For example, adoption of policies that improve stock market's transparency and programs that promote financial literacy can mitigate the negative effects of low trust and induce stockholding among wealthier households in European countries where stockholding is not widespread. 


\section{References}

Brambor, Thomas, William Clark, and Matt Golder (2005). "Understanding interaction models: Improving Empirical Analysis", Political Analysis, 13, pp. 1-20.

Burbidge, John B., Lonnie Magee, and A. Leslie Robb (1988). "Alternative transformations to handle extreme values of the dependent variable", Journal of the American Statistical Association 83, 123-127.

Calvó-Armengol, Antoni, Eleonora Patacchini, and Yves Zenou (2005). "Peer Effects and Social Networks in Education and Crime". CEPR Discussion Paper No. 5244.

Campbell, John Y. (2006). "Household Finance". The Journal of Finance 61(4), pp. 1553 1604.

Christelis, Dimitris, Dimitris Georgarakos, and Michael Haliassos (2008). "Economic Integration and Mature Portfolios". Center for Financial Studies, WP 2008/05.

Christelis, Dimitris, Tullio Jappelli, and Mario Padula (2009). "Cognitive Abilities and Portfolio Choice". forthcoming in European Economic Review.

Duflo, Esther and Emmanuel Saez (2002). "Participation and Investment Decisions in a Retirement Plan: the Influence of Colleagues' Choices". Journal of Public Economics 85(1), pp. 121-148.

Durlauf, Steven N. and Marcel Fafchamps (2004). "Social Capital". NBER Working Paper No. W10485.

Fehr, Ernst (2009). "On The Economics and Biology of Trust". Journal of the European Economic Association 7(2-3), pp. 235-266.

Granovetter, Mark S. (1973). "The Strength of Weak Ties". American Journal of Sociology 78(6), pp. 1360- 1380.

Guiso, Luigi, Michael Haliassos, and Tullio Jappelli (2002). Household Portfolios. MIT: Cambridge, UK.

Guiso, Luigi, Paola Sapienza, and Luigi Zingales (2003). "People's Opium. The Economic Effects of Religion". Journal of Monetary Economics 50, pp. 225-282.

Guiso, Luigi, Paola Sapienza, and Luigi Zingales (2004). "The Role of Social Capital in Financial Development". American Economic Review 94, pp. 526-556.

Guiso, Luigi, Paola Sapienza, and Luigi Zingales (2008). "Trusting the Stock Market". The Journal of Finance 63(6), pp. 2557-2600.

Guiso, Luigi, and Tullio Jappelli (2005). “Awareness and Stock Market Participation”. Review of Finance 9, pp. 537-567.

Haliassos, Michael and Carol C. Bertaut (1995). "Why Do So Few Hold Stocks?". The Economic Journal 105(432), pp. 1110-1129.

Hong, Harrison, Jeffrey D. Kubik, and Jeremy C. Stein (2004). "Social Interaction and StockMarket Participation". The Journal of Finance 59(1), pp. 137-163.

Islam, M. Kamrul, Juan Merlo, Ichiro Kawachi, Martin Lindström, and Ulf G. Gerdtham (2006). "Social Capital and Health: Does Egalitarianism Matter? A Literature Review". International Journal for Equity in Health 5(3), pp. 1-28.

Jackson, Matthew O. (2006). "The Economics of Social Networks" in Advances in Economics \& Econometrics, Theory \& Applications: Ninth World Congress of the Econometric Society, ed. by R. Blundell, W. Newey, and T. Persson. Cambridge University Press, Chap. 1, Vol. 1, pp. 1-56.

Knack, Stephen and Philip Keefer (1997). "Does Social Capital Have an Economic Payoff? A Cross-Country Investigation". The Quarterly Journal of Economics 112(4), pp. 1251-1288. 
La Porta, Rafael, Florencio Lopez-De-Silanes, and Andrei Shleifer (2006). "What Works in Securities Laws?". The Journal of Finance 61(1), pp. 1-32.

Madrian, Brigitte and Dennis Shea (2000). "Peer effects and savings behavior in employersponsored savings plans". University of Chicago working paper

Mankiw, N. Gregory and Stephen P. Zeldes (1991). "The Consumption of Stockholders and Nonstockholders". Journal of Financial Economics 29(1), pp. 97-112.

Manski, Charles F. (1993). "Identification of Endogenous Social Effects: The Reflection Problem". Review of Economic Studies 60, pp.531-542

Moulton, Brent R. (1990). "An Illustration of a Pitfall in Estimating the Effects of Aggregate Variables on Micro Unit". Review of Economics and Statistics 72, pp. 334-38.

Newton, Kenneth (2001). “Trust, Social Capital, Civil Society, and Democracy”. International Political Science Review 22(2), pp. 210-214.

Putnam, Robert D. (2000). "Bowling Alone: The Collapse and Revival of American Community". New York: Simon \& Schuster.

Putman, Robert D., Robert Leonardi, and Raffaella Y. Nanetti (1993). "Making Democracy Work, Civic Traditions in Modern Italy". Princeton, NJ: Princeton University Press.

Rosen, Harvey S. and Stephen Wu (2004). "Portfolio Choice and Health Status". Journal of Financial Economics 72(3), pp 457-484.

Rostila, Mikael (2007). "Social Capital and Health in European Welfare Regimes: a Multilevel Approach". Journal of European Social Policy 17(3), pp. 223-239.

Rubin, Donald B. (1987). "Multiple Imputation for Nonresponse in Surveys". J. Wiley \& Sons, New York.

Vega-Redondo, Fernando (2006). "Building up Social Capital in a Changing World”. Journal of Economics Dynamics and Control 30, pp. 2305-2338. 


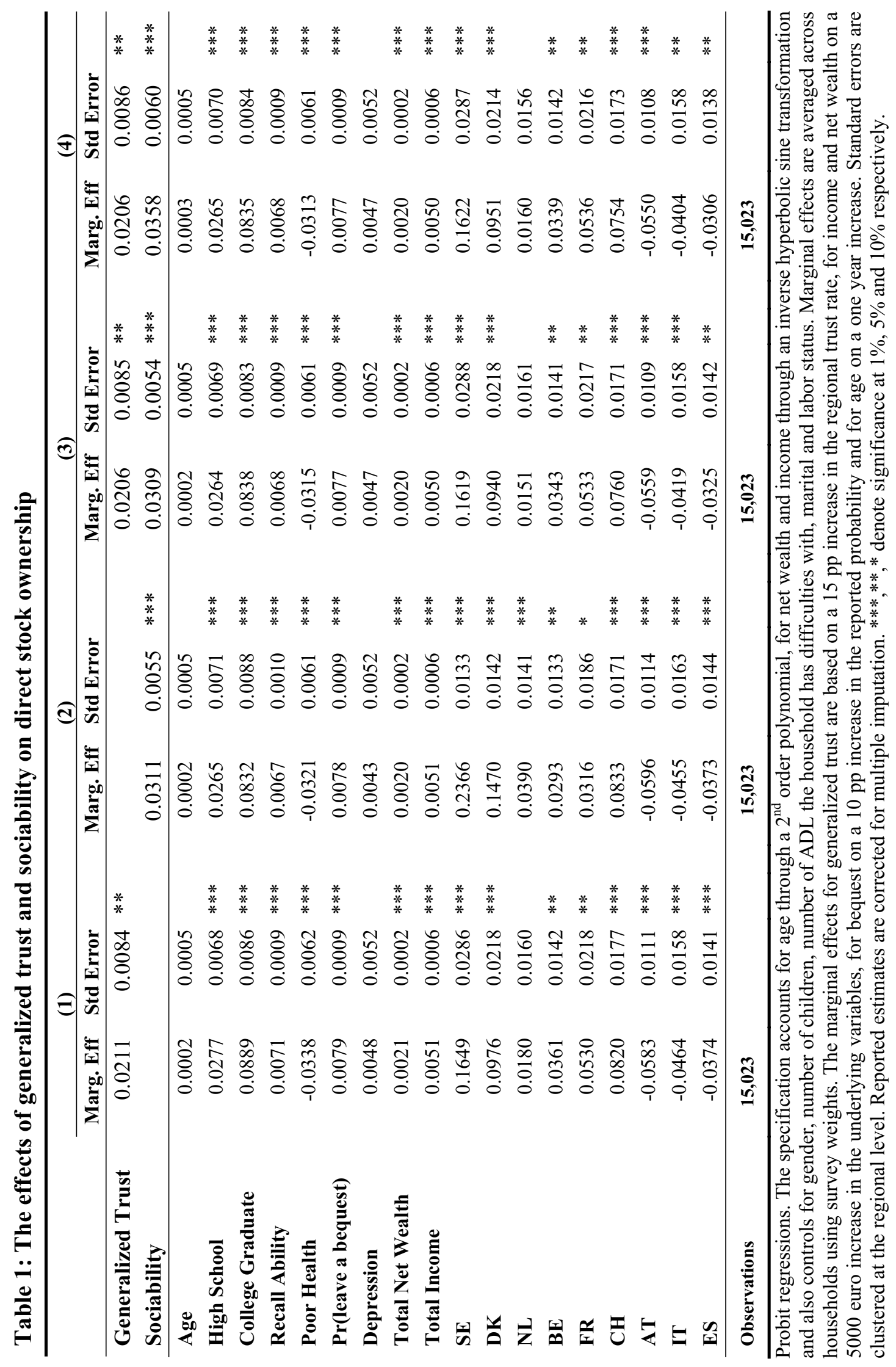




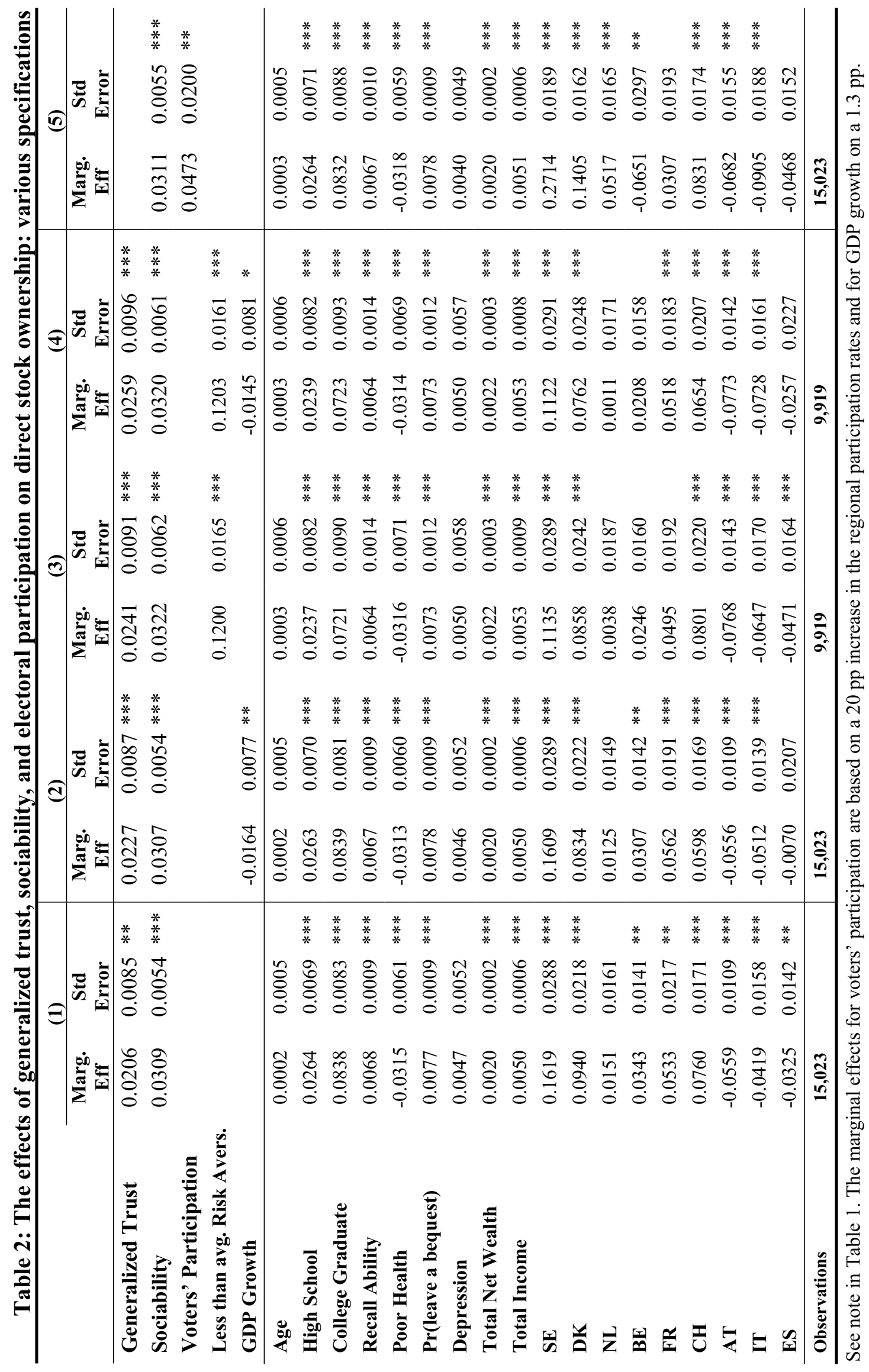


Table 3: Trust in financial institutions and assessment of financial services by country

\begin{tabular}{lccccc}
\hline & $\begin{array}{c}\text { Trust in } \\
\text { Financial } \\
\text { Institutions }\end{array}$ & $\begin{array}{c}\text { Informal } \\
\text { Borrowing }\end{array}$ & $\begin{array}{c}\text { Easy Access } \\
\text { to Formal } \\
\text { Credit }\end{array}$ & $\begin{array}{c}\text { Very Difficult } \\
\text { to Change } \\
\text { Banks }\end{array}$ & $\begin{array}{c}\text { Use of } \\
\text { Internet } \\
\text { Banking }\end{array}$ \\
\hline SE & 0.57 & 0.91 & 0.16 & 0.02 & 0.47 \\
DK & 0.78 & 0.88 & 0.23 & 0.03 & 0.42 \\
DE & 0.65 & 0.66 & 0.17 & 0.04 & 0.14 \\
NL & 0.60 & 0.88 & 0.21 & 0.08 & 0.34 \\
BE & 0.64 & 0.66 & 0.20 & 0.08 & 0.15 \\
FR & 0.53 & 0.79 & 0.32 & 0.06 & 0.12 \\
AT & 0.75 & 0.76 & 0.27 & 0.06 & 0.19 \\
IT & 0.42 & 0.82 & 0.09 & 0.06 & 0.07 \\
ES & 0.46 & 0.91 & 0.11 & 0.05 & 0.08 \\
\hline Weit
\end{tabular}

Weighted statistics. Data on Informal Borrowing is from Eurobarometer 56.1 (2001). All other data is from Eurobarometer 60.2 (2003). 


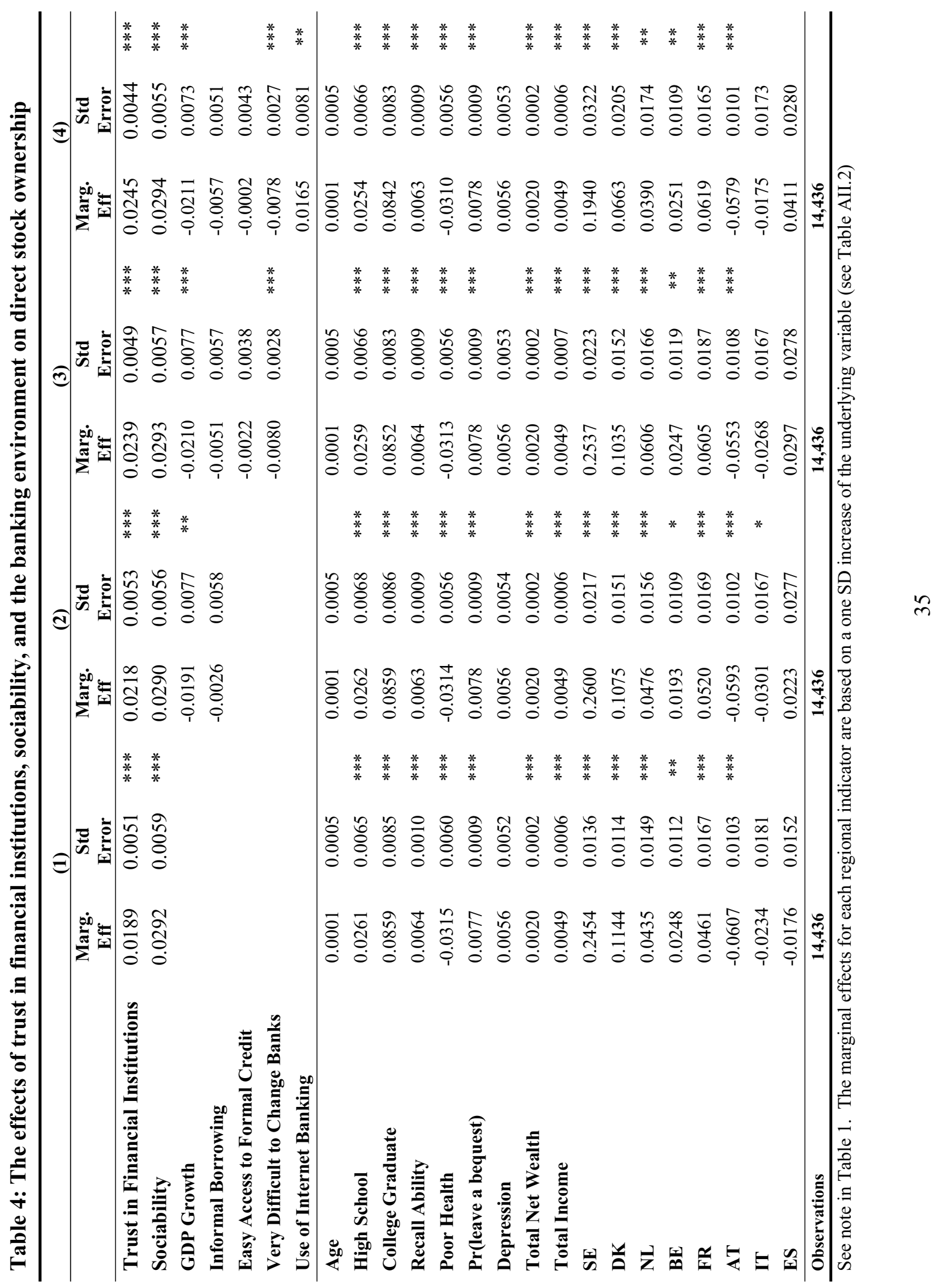


Table 5: The effects of generalized trust and sociability on direct stock ownership across countries with high, medium, low stock market participation and by above/ below median household wealth

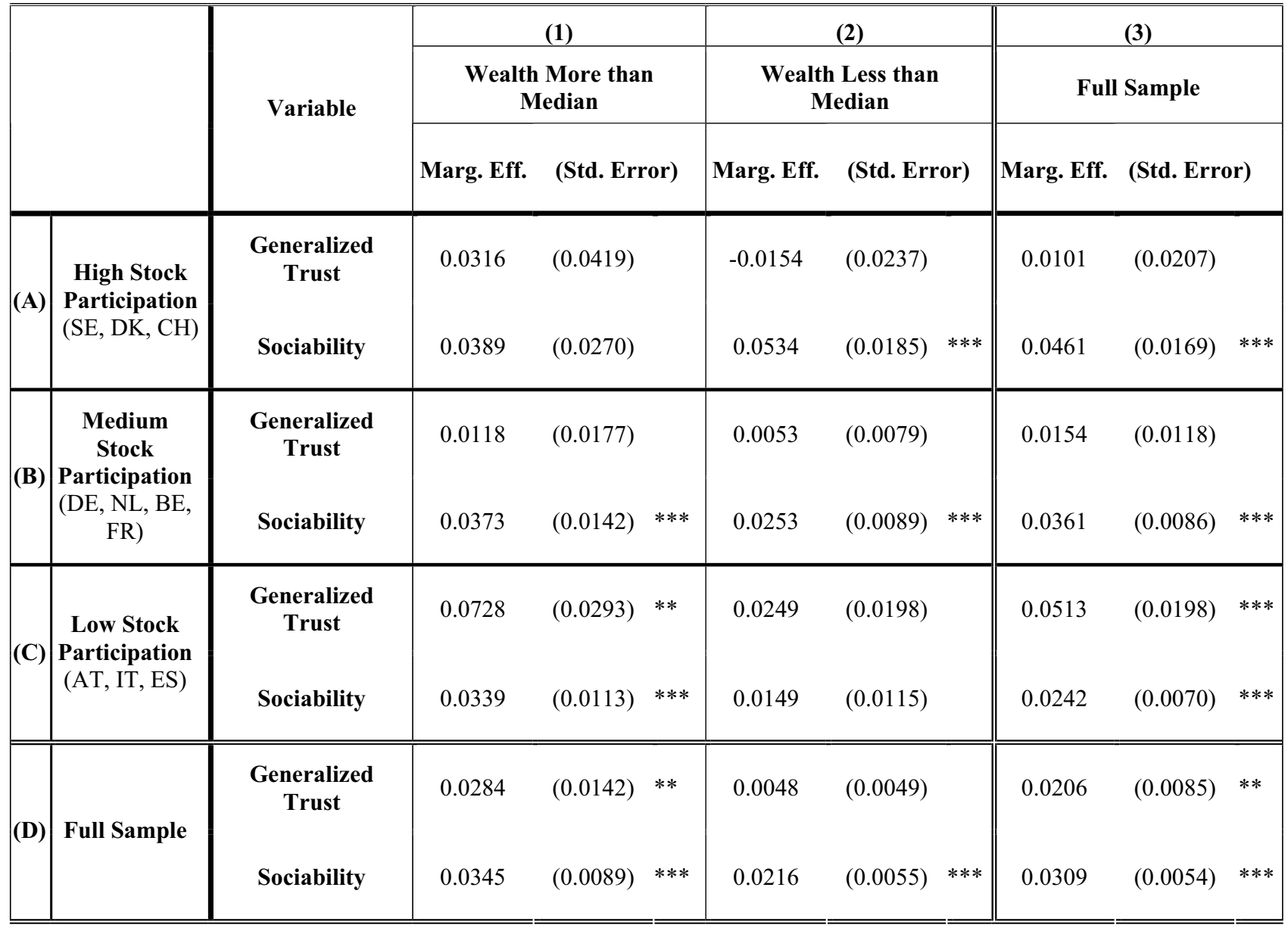

Each model conditions on the same set of regressors as the baseline model 3 in Table 1 including the relevant country dummies. See note in Table 1. 
Figure 1: Direct stock ownership rates by country

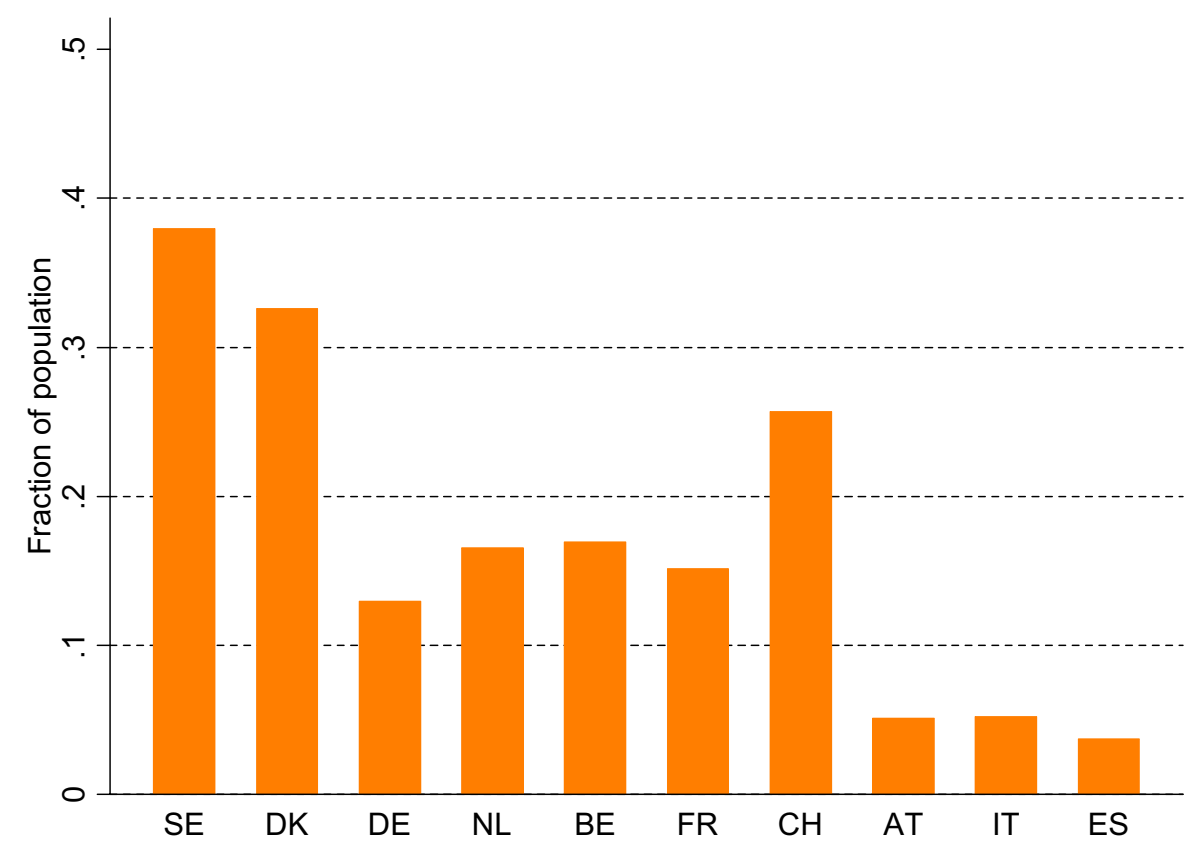

Weighted data from SHARE 2004. 
Figure 2: Average Sociability and Generalized Trust by country

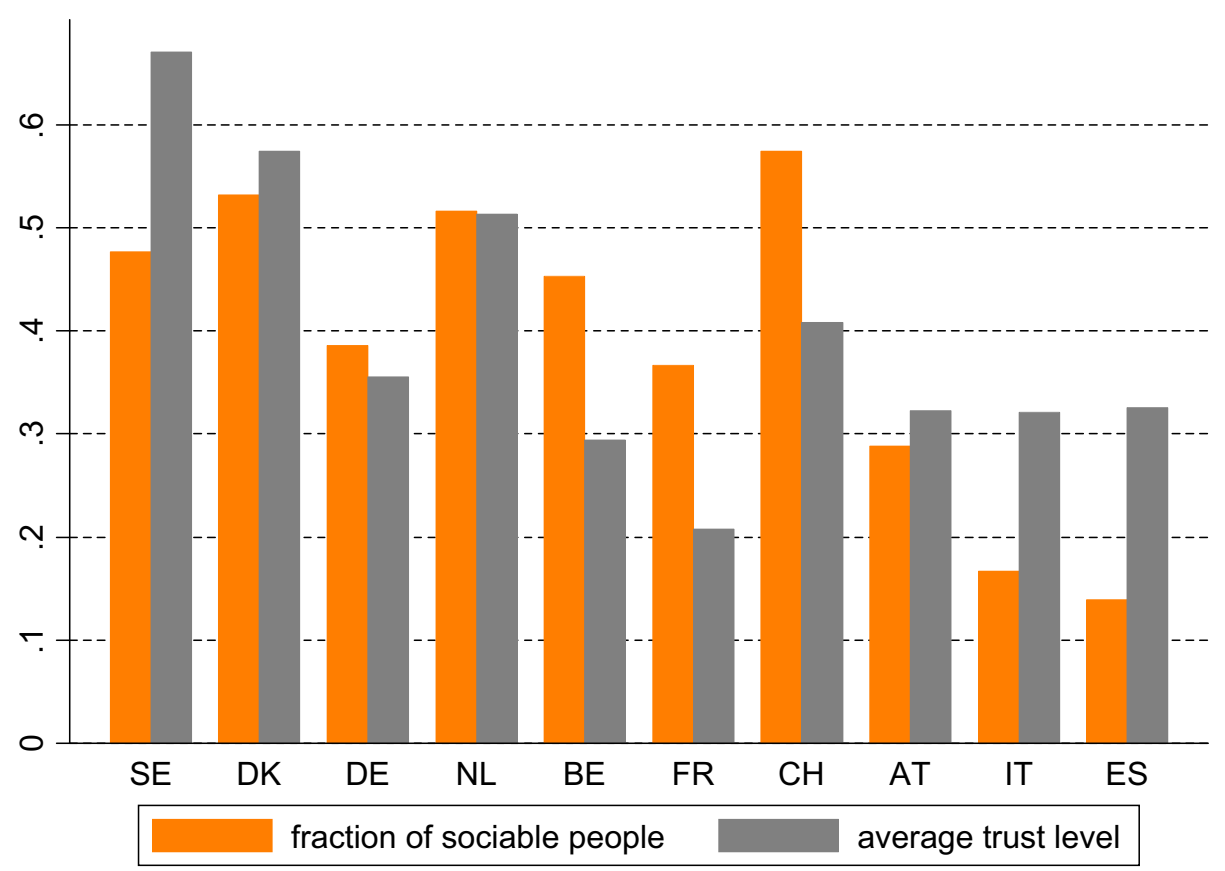

Weighted data on generalized trust from WVS (1990-2005) and on sociability from SHARE 2004. 
Figure 3: Generalized Trust rates by region

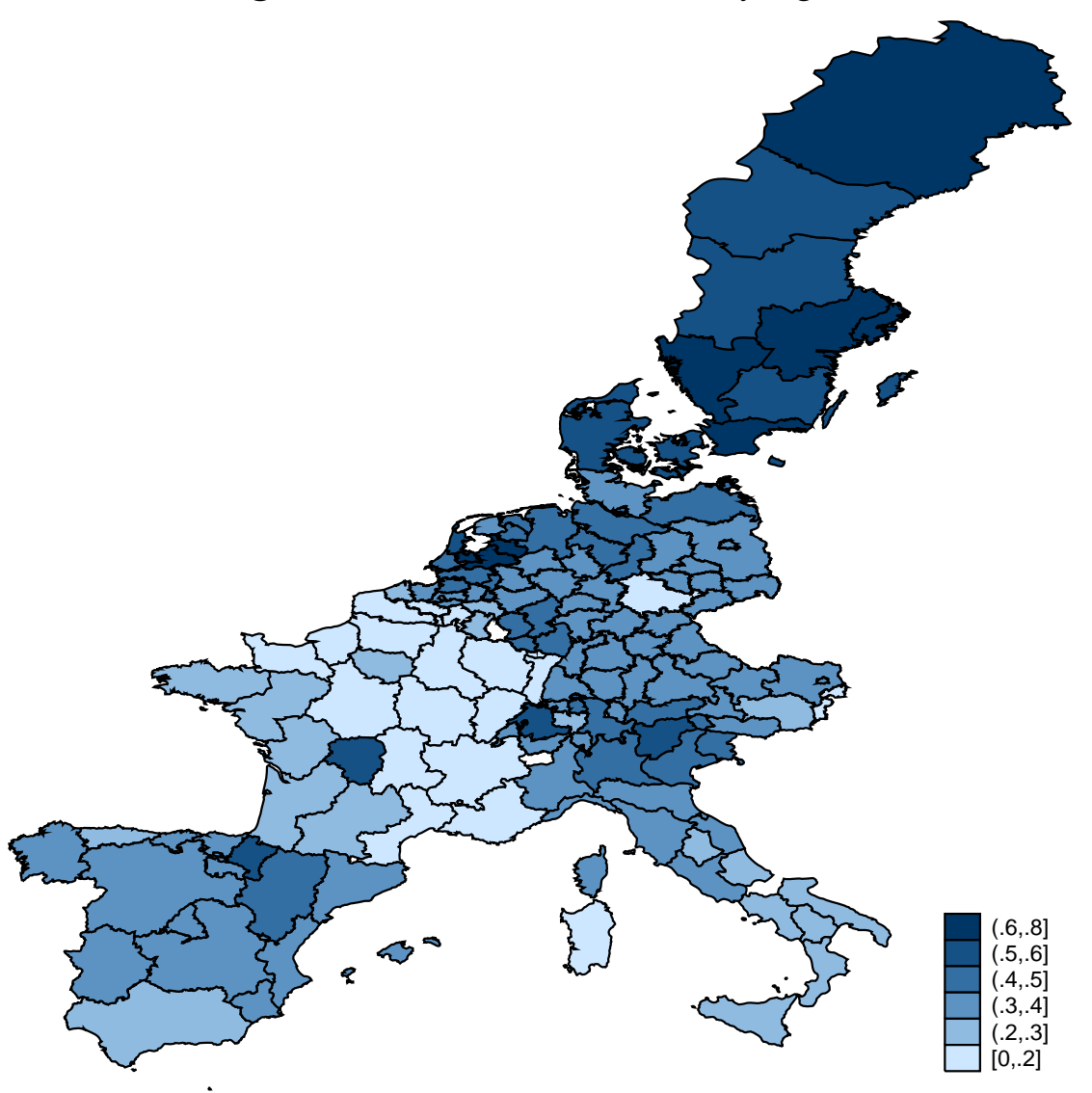

Weighted data from WVS (1990-2005). 
Figure 4: Direct stock ownership rates across countries with high, medium, low stock market participation and by above/ below median household wealth

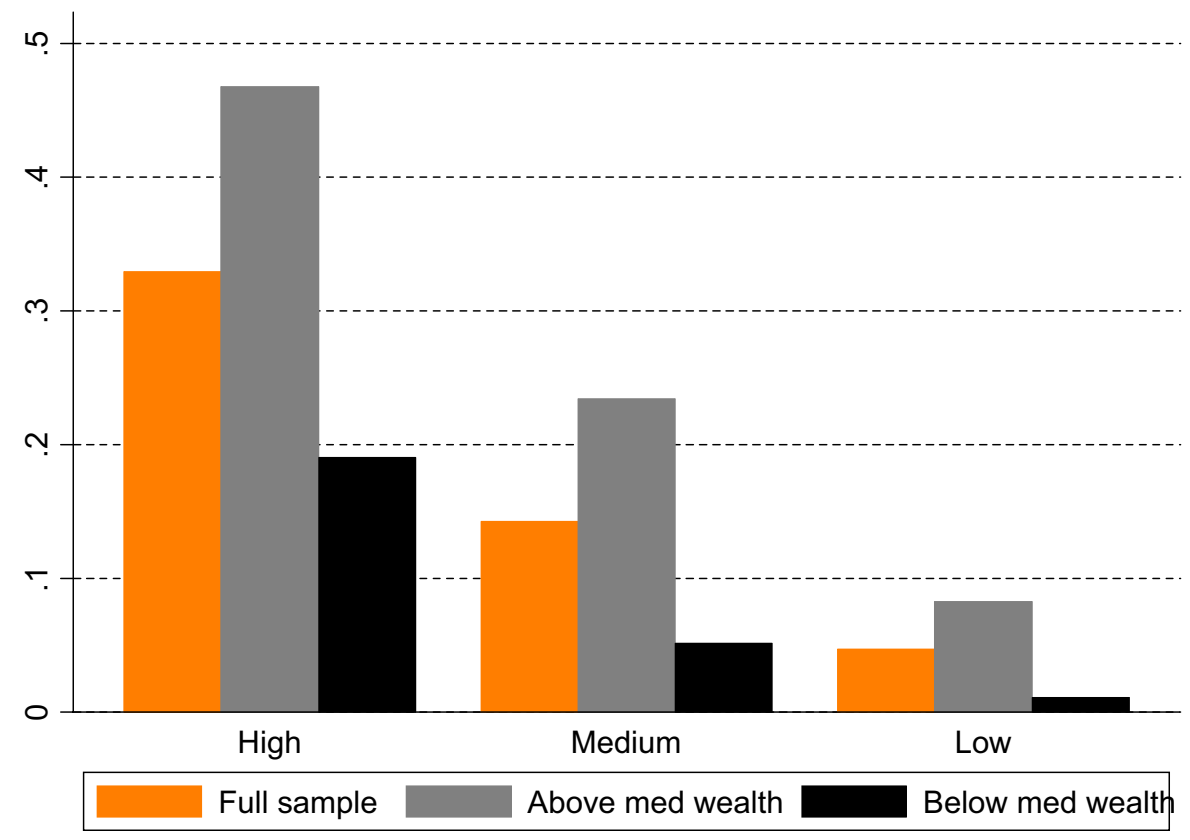

Weighted data from SHARE 2004. Countries are divided according to the average stock market participation into 'high' (SE, DK, CH), 'medium' (DE, NL, BE, FR) and 'low' (AT, IT, ES). 


\section{Appendix I: proof of proposition 1}

Condition (10) can be rewritten as

$$
\begin{aligned}
\left.\left.(1-\bar{p}) U\left[\hat{\alpha}(\underline{W}-f(\underline{\pi}))+(1-\alpha) r_{f}(\underline{W}-f(\underline{\pi}))\right]+\bar{p} U \mid(1-\alpha) r_{f}(\underline{W}-f(\underline{\pi}))\right]-U \mid r_{f} \underline{W}\right]=0 \\
F[\bar{p}, \underline{\pi}, \alpha]=0
\end{aligned}
$$

Equation (11) defines two implicit conditional relationships:

$$
\begin{aligned}
& \bar{p}=g(\underline{\pi} \mid \alpha, \underline{W}) \\
& \bar{p}=f\left(\underline{W} \mid \alpha^{*}, \underline{\pi}\right)
\end{aligned}
$$

The relation $\bar{p}=g(\underline{\pi} \mid \alpha, \underline{W})$ can be described using the implicit function theorem which states that $\frac{d p}{d \pi}=-\frac{\partial F / \partial \pi}{\partial F / \partial p}$.

Recall that $\alpha \hat{r}(W-f)+(1-\alpha) r_{f}(W-f(\underline{\pi}))=A$ and $(1-\alpha) r_{f}(W-f(\underline{\pi}))=B$ : by equation (9), $A>B$. Thus, given that $U$ is monotonically non decreasing, $A>B \Rightarrow U[A] \geq U[B]$ and $d U[x] / d x \geq 0$. We can sign the following partial derivatives:

$$
\begin{aligned}
& \partial F / \partial p=-U[A]+U[B]<0 \\
& \partial F / \partial \pi=-(1-\bar{p}) \frac{d U}{d A}\left(\alpha \hat{r} \frac{\partial f}{\partial \pi}+(1-\alpha) r_{f} \frac{\partial f}{\partial \pi}\right)>0
\end{aligned}
$$

Therefore $\frac{d p}{d \pi}>0$, which means that given $\alpha>0$ and $W=\underline{W}$, the level of trust $(1-\bar{p})$ and minimum sociability level $\underline{\pi}$ which trigger participation are inversely related.

Given that $\bar{p}=f\left(\underline{W} \mid \alpha^{*}, \underline{\pi}\right)$, once sociability is fixed the relation between trust and wealth is exactly the same to that described by GSZ and thus their proposition 4 holds. 


\section{Appendix II: data descriptions}

As discussed in section 4, we first compute the generalized trust indicator as the region-level average of responses to the trust question contained in the WVS. We then assign the relevant average to every SHARE respondent who lives in the same region. Regional information on SHARE is not homogenous across countries due to different disclosure rules in Europe: for most countries in the sample it corresponds to the NUTS2 statistical regions (as defined by Eurostat), to the NUTS3 regions in Denmark, Sweden and Spain, while in France and Germany the regional disaggregation is at NUTS1 level. As a general rule we use the most disaggregated regional definition available in SHARE to merge with regional data collected from other sources. In the same way we merge SHARE with Eurobarometer data that offer information on a specific measure of trust and other features of the banking environment.

We have to drop Greece from our analysis, since it was not feasible to match all NUTS2 Greek regions in SHARE with regions available in WVS. ${ }^{35}$ Ekinci, Kalemli-Ozcan and Sorensen (2007), who use regional data from WVS to examine financial integration among European regions, also exclude Greece on the ground that it is less economically developed compared to other countries in the sample and thus has the character of an outlier. The WVS did not take place in the same year in all the countries we are interested in. Moreover, the number of waves available for each country varies as well. WVS took place just once in the last 25 years in Switzerland, while there were four surveys in Spain during the same period. In order to facilitate a significant number of observations in each region that we use to merge with SHARE, we pooled data from all available WVS waves since 1990. This is also consistent with the notion that generalized trust is typically deeply rooted and associated with an area's history, norms, and traditions and that it hardly changes with time.

On the other hand, Eurobarometer data with relevant information have been collected only in the end of 2003 (module 60.2), offering current information on certain features of the banking environment faced by households in the 2004 SHARE sample. ${ }^{36}$ In merging with SHARE we ignore the few cases of regions in which data have not been collected or the

\footnotetext{
${ }^{35}$ WVS interviews households from specific sub-areas within the NUTS2 Greek regions. We have also experimented by projecting data from these specific sub-areas to the NUTS2 regional level and then merging with SHARE data in order to include Greece in our regressions. This left our main findings qualitatively unchanged.

${ }^{36}$ Information on informal borrowing is only collected in the end of 2001, thus we have to use an earlier Eurobarometer module (56.1) in order to recover it.
} 
number of respondents is very small (less than 50). This results in a relatively smaller sample size (of roughly 600 households) in the estimation that employs merged SHARE and Eurobarometer data (Table 4) compared to the one used in the baseline specification (Table 1).

Data on regional GDP growth rates were collected from Swiss statistics for Switzerland and from Eurostat for all the other countries in the sample. Data on voters' participation rates by region in 2004 EU Elections (and 2003 National Elections for Switzerland) were collected from various national statistical sources. Finally, Tables AII.1 and AII.2 provide summary statistics as well as a detailed description of all variables used in the empirical analysis. 


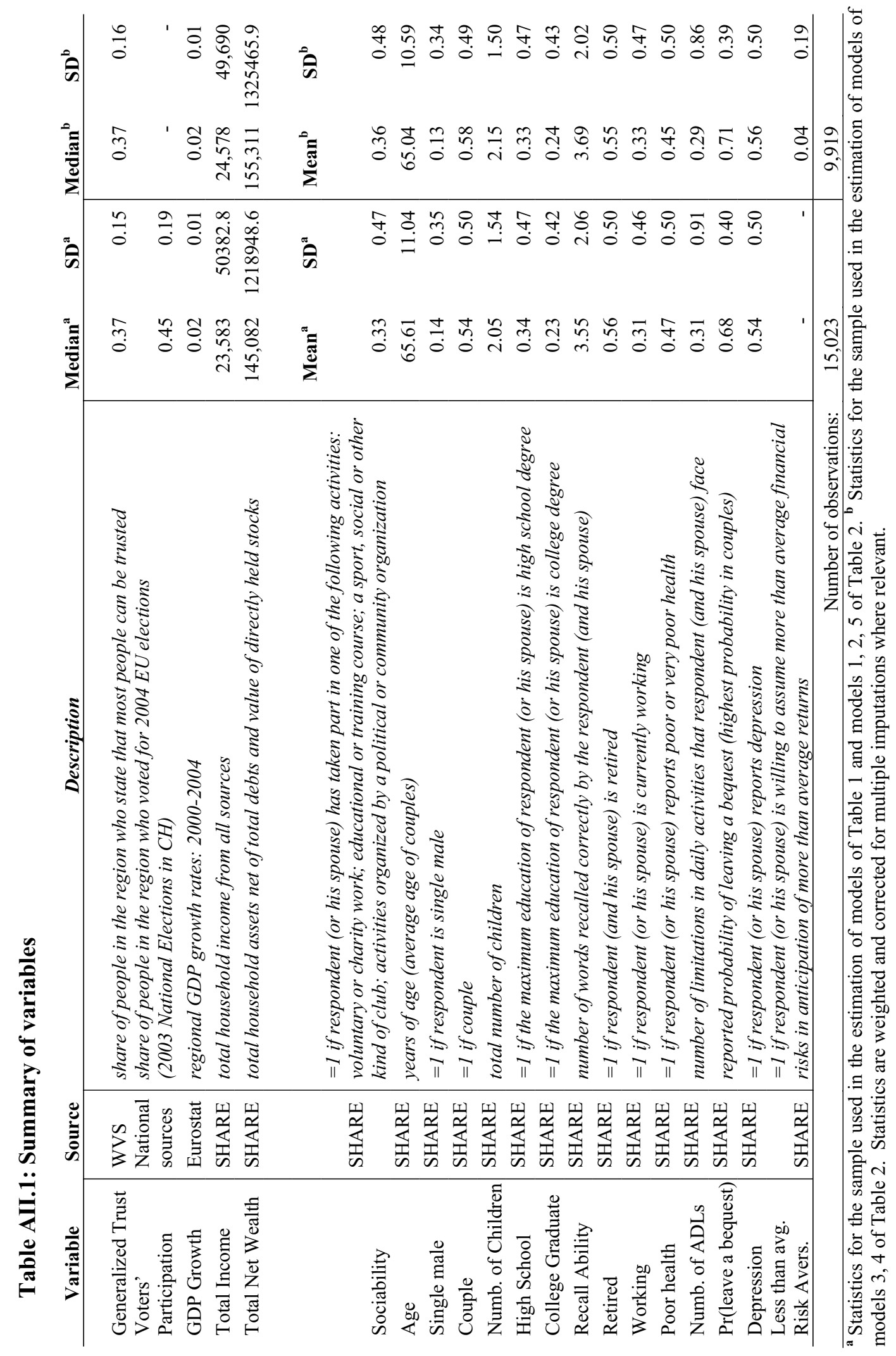




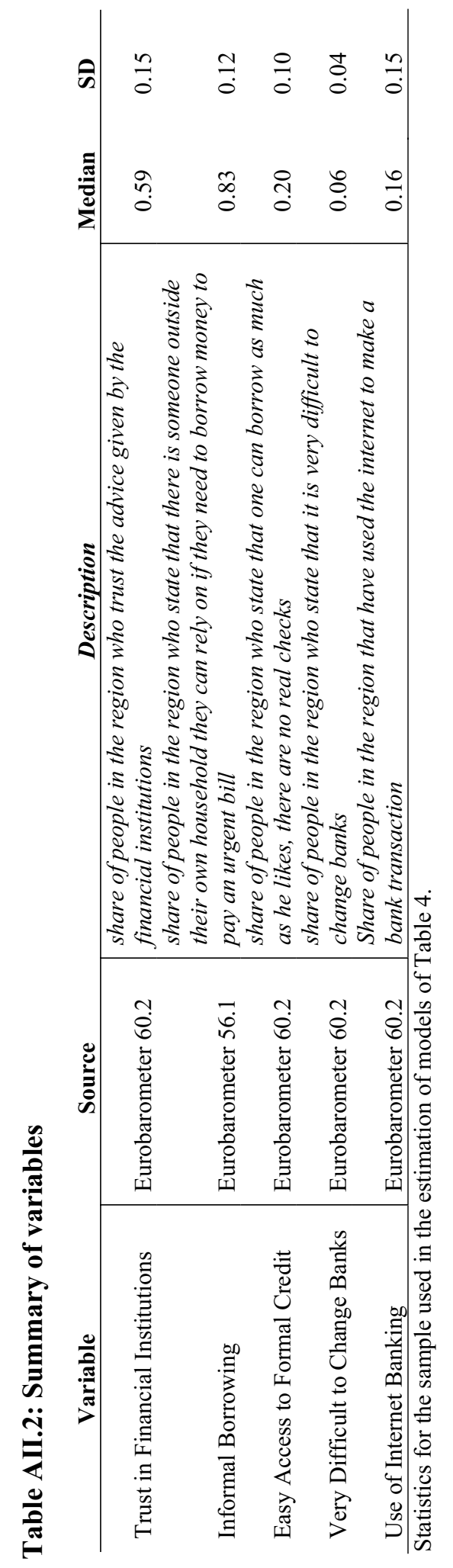




\section{CFS Working Paper Series:}

\begin{tabular}{lll} 
No. & Author(s) & Title \\
\hline $2009 / 28$ & $\begin{array}{l}\text { Dimitris Christelis } \\
\text { Dimitris Georgarakos }\end{array}$ & $\begin{array}{l}\text { Investing at Home and Abroad: Different } \\
\text { Costs, Different People? }\end{array}$ \\
$2009 / 27$ & Erik Theissen & $\begin{array}{l}\text { Price Discovery in Spot and Futures Markets: } \\
\text { A Reconsideration }\end{array}$
\end{tabular}

2009/26 Volker Wieland

Fiscal stimulus and the promise of future spending cuts: A comment

$\begin{array}{ll}2009 / 25 & \begin{array}{l}\text { Tobias Cwik } \\ \text { Volker Wieland }\end{array}\end{array}$

2009/24 Otmar Issing

2009/23 Nikolaus Hautsch Ruihong Huang

2009/22 Christian Laux Christian Leuz

2009/21 John B. Taylor Volker Wieland

2009/20 Nikolaus Hautsch

Lada M. Kyj

Roel C.A. Oomen

2009/19 Guenter W. Beck Volker Wieland
Keynesian government spending multipliers and spillovers in the euro area

Politischer Wille oder ökonomisches Gesetz?

- Einige Anmerkungen zu einem großen

Thema -

The Market Impact of a Limit Order

Did Fair-Value Accounting Contribute to the Financial Crisis?

Surprising Comparative Properties of Monetary Models: Results from a New Data Base

A blocking and regularization approach to high dimensional realized covariance estimation

Money in Monetary Policy Design: Monetary Cross-Checking in the New-Keynesian Model

Copies of working papers can be downloaded at http://www.ifk-cfs.de 